TKYNT-08-27, YITP-08-86

\title{
Lattice study of flavor SU(3) breaking in hyperon beta decay
}

\author{
Shoichi Sasak* \\ Department of Physics, The University of Tokyo, \\ Hongo 7-3-1, Tokyo 113-0033, Japan \\ Takeshi Yamazak $\dagger$ \\ Physics Department, University of Connecticut, \\ Storrs, Connecticut 06269-3046, USA and \\ Yukawa Institute for Theoretical Physics, Kyoto University, \\ Kitashirakawa-Oiwakecho, Sakyo, Kyoto 606-8502, Japan
}

(Dated: October 25, 2018)

\begin{abstract}
We present a quenched lattice calculation of all six form factors: vector $\left[f_{1}\left(q^{2}\right)\right]$, weak magnetism $\left[f_{2}\left(q^{2}\right)\right]$, induced scalar $\left[f_{3}\left(q^{2}\right)\right]$, axial-vector $\left[g_{1}\left(q^{2}\right)\right]$, weak electricity $\left[g_{2}\left(q^{2}\right)\right]$, and induce pseudoscalar $\left[g_{3}\left(q^{2}\right)\right]$ form factors, in hyperon semileptonic decay $\Xi^{0} \rightarrow \Sigma^{+} l \bar{\nu}$ using domain wall fermions. The $q^{2}$ dependences of all form factors in the relatively low $q^{2}$ region are examined in order to evaluate their values at zero momentum transfer. The $\Xi^{0} \rightarrow \Sigma^{+}$transition is highly sensitive to flavor $S U(3)$ breaking since this decay corresponds to the direct analogue of neutron beta decay under the exchange of the down quark with the strange quark. The pattern of flavor $S U(3)$ breaking effects in the hyperon beta decay is easily exposed in a comparison to results for neutron beta decay. We measure $S U(3)$-breaking corrections to $f_{1}(0), f_{2}(0) / f_{1}(0)$ and $g_{1}(0) / f_{1}(0)$. A sign of the leading order corrections, of which the size is less than a few $\%$, on $f_{1}(0)$ is likely negative, while $f_{2}(0) / f_{1}(0)$ and $g_{1}(0) / f_{1}(0)$ receive positive corrections of order $16 \%$ and $5 \%$ respectively. The observed pattern of the deviation from the values in the exact $S U(3)$ limit does not support some of model estimates. We show that there are nonzero second-class form factors in the $\Xi^{0} \rightarrow \Sigma^{+}$ decay, measuring $f_{3}(0) / f_{1}(0)=0.14(9)$ and $g_{2}(0) / g_{1}(0)=0.68(18)$, which are comparable to the size of first-order $S U(3)$ breaking. It is also found that the $S U(3)$ breaking effect on $g_{3}(0) / g_{1}(0)$ agrees with the prediction of the generalized pion-pole dominance.
\end{abstract}

\section{INTRODUCTION}

The latest lattice calculations of $K \rightarrow \pi l \nu\left(K_{l 3}\right)$ semileptonic decays have been greatly developed with high precision [1, 2, 3]. The results for the vector form factor $f_{+}(0)$ of $K_{l 3}$ decays, which deviates from unity due to flavor $S U(3)$ breaking, can be used to provide a very precise determination of the element $V_{u s}$ of the Cabibbo-KobayashiMaskawa (CKM) matrix [4, 5]. Those first-principles calculations contribute greatly to a stringent test of the CKM unitary through the first row relation $\left|V_{u d}\right|^{2}+\left|V_{u s}\right|^{2}+\left|V_{u b}\right|^{2}=1[6]$. On the other hand, $\Delta S=1$ semileptonic hyperon decays provide alternative determinations of $\left|V_{u s}\right|$ [7]. The consistency of the values of $\left|V_{u s}\right|$ determined from different experiments needs to be confirmed. As we will explain later, however, the determination of $\left|V_{u s}\right|$ from the hyperon decays should be affected by larger theoretical uncertainties than those of $K_{l 3}$ decays [8]. This is simply because the lack of a reliable theoretical calculation of the leading symmetry-breaking corrections in the hyperon beta decays. Indeed, the conventional Cabibbo model [5], where flavor $S U(3)$ breaking effects are ignored, is commonly used for the analysis of the hyperon beta-decay data $[9]$.

An essential difference from the case of $K_{l 3}$ decays is that the axial-vector current also contributes to the transition in the hyperon beta decays. Thus, the precise determination of $\left|V_{u s}\right|$ in the hyperon beta decays requires information of the ratio of the axial-vector to vector form factors $g_{1}(0) / f_{1}(0)$ in addition to the vector form factor $f_{1}(0)$ [9]. Here we recall that the various ratios $g_{1}(0) / f_{1}(0)$ in the hyperon beta decays also provide vital information to analysis of strange quark spin fraction of the proton spin, together with the polarized deep inelastic scattering data [10]. However, such analysis heavily relies on the Cabibbo model. The flavor $S U(3)$ breaking introduces the systematic uncertainty on the strange quark contribution to the proton spin. This issue is still under debate [11]. Recently, a new analysis, where the first-order corrections of both $S U(3)$ and $S U(2)$ symmetry breaking are properly taken into

*Electronic address: ssasaki@phys.s.u-tokyo.ac.jp

$\dagger$ Electronic address: tyamazak@yukawa.kyoto-u.ac.jp 
account within the Cabibbo model, was proposed by Yamanishi [12]. The author has reported that flavor breaking effects significantly affect the evaluation of the amount of spin carried by the strange quarks inside the proton [12].

In the hyperon decays, a model independent evaluation of $S U(3)$-breaking corrections is highly demanded for both the CKM unitary test and the proton spin problem. The weak matrix element of the hyperon beta decays can be calculated with high accuracy from first principles using the techniques of lattice QCD, similar to what is achieved in the case of $K_{l 3}$ decays [1, 2, 3]. There is a single lattice study to be completed for a specific hyperon decay, $\Sigma^{-} \rightarrow n l \bar{\nu}$. The lattice simulation have been performed by Guadagnoli et al. with $\mathcal{O}(a)$-improved Wilson fermions in the quenched approximation [13] 57. As we are interested primarily in flavor $S U(3)$ breaking effects not in the absolute values of each form factor, we choose a different hyperon decay, $\Xi^{0} \rightarrow \Sigma^{+} l \bar{\nu}$ in this study. This is simply because the $\Xi^{0} \rightarrow \Sigma^{+}$ transition is the direct analogue of neutron beta decay under the exchange of the down quark with the strange quark. The flavor $S U$ (3)-breaking can be easily exposed through a comparison with results of neutron beta decay. In Ref. [14], for the axial-vector coupling of neutron beta decay, $g_{1}(0)=1.212(27)$ in the chiral limit is obtained from quenched lattice QCD calculations with domain wall fermions (DWFs). It underestimates the experimental value of 1.2695(29) by less than 5\%. Other relevant weak form factors have been subsequently investigated in Ref. [15]. We naturally extend the quenched DWF calculation for investigating $S U(3)$-breaking corrections to the hyperon semileptonic decay form factors.

Our paper is organized as follows. In Sec. II , we first present a brief introduction of the hyperon beta decays. In Sec. III details of our Monte Carlo simulations and some basic results are given. We also describe the lattice method for calculating the baryon beta-decay form factors. Section IV is devoted to our determination of the scalar form factor $f_{S}\left(q^{2}\right)$, which will be defined in the next section, at finite momentum transfer. We describe the interpolation of the form factor to zero momentum transfer and the chiral extrapolation in order to evaluate the value of $f_{1}(0)$ at the physical point. We also present our estimate of $\left|V_{u s}\right|$. Section $\mathbf{V}$ gives the result of the ratio of $g_{1}(0) / f_{1}(0)$ at the physical point. We then discuss flavor $S U(3)$-breaking effects appeared in $g_{1}(0) / f_{1}(0)$ for the $\Xi^{0} \rightarrow \Sigma^{+}$transition in comparison to neutron beta decay. The results for the other form factors including the second-class form factors $f_{3}$ and $g_{2}$ are presented in Sec. VI. Finally, in Sec. VII, we summarize our results and conclusions.

\section{HYPERON BETA DECAYS}

The general form of the baryon matrix element for semileptonic decays $B \rightarrow b l \bar{\nu}$ is given by both the vector and axial-vector transitions:

$$
\left\langle b\left(p^{\prime}\right)\left|V_{\alpha}(x)+A_{\alpha}(x)\right| B(p)\right\rangle=\bar{u}_{b}\left(p^{\prime}\right)\left(\mathcal{O}_{\alpha}^{V}(q)+\mathcal{O}_{\alpha}^{A}(q)\right) u_{B}(p) e^{i q \cdot x},
$$

where $q \equiv p-p^{\prime}$ is the momentum transfer between the initial state $(B)$ and the final state $(b)$ which belong to the lightest $J^{P}=1 / 2^{+} S U(3)$ octet of baryons $(p, n, \Lambda, \Sigma, \Xi)$. The vector and axial-vector currents are defined as $V_{\alpha}(x)=$ $\bar{u}(x) \gamma_{\alpha} d(x)$ and $A_{\alpha}(x)=\bar{u}(x) \gamma_{\alpha} \gamma_{5} d(x)$ for $\Delta S=0$ decays, and $V_{\alpha}(x)=\bar{u}(x) \gamma_{\alpha} s(x)$ and $A_{\alpha}(x)=\bar{u}(x) \gamma_{\alpha} \gamma_{5} s(x)$ for $\Delta S=1$ decays. Six form factors are needed to describe the hyperon beta decays: the vector $\left(f_{1}\right)$, weak magnetism $\left(f_{2}\right)$ and induced scalar $\left(f_{3}\right)$ form factors for the vector current,

$$
\mathcal{O}_{\alpha}^{V}(q)=\gamma_{\alpha} f_{1}^{B \rightarrow b}\left(q^{2}\right)+\sigma_{\alpha \beta} q_{\beta} \frac{f_{2}^{B \rightarrow b}\left(q^{2}\right)}{M_{B}+M_{b}}+i q_{\alpha} \frac{f_{3}^{B \rightarrow b}\left(q^{2}\right)}{M_{B}+M_{b}}
$$

and the axial-vector $\left(g_{1}\right)$, weak electricity $\left(g_{2}\right)$ and induced pseudo-scalar $\left(g_{3}\right)$ from factors for the axial current,

$$
\mathcal{O}_{\alpha}^{A}(q)=\gamma_{\alpha} \gamma_{5} g_{1}^{B \rightarrow b}\left(q^{2}\right)+\sigma_{\alpha \beta} q_{\beta} \gamma_{5} \frac{g_{2}^{B \rightarrow b}\left(q^{2}\right)}{M_{B}+M_{b}}+i q_{\alpha} \gamma_{5} \frac{g_{3}^{B \rightarrow b}\left(q^{2}\right)}{M_{B}+M_{b}},
$$

which are here given in the Euclidean metric convention (we have defined $\sigma_{\alpha \beta}=\frac{1}{2 i}\left[\gamma_{\alpha}, \gamma_{\beta}\right]$.) [58]. Here, $M_{B}\left(M_{b}\right)$ denotes the rest mass of the initial (final) state. Note that although the sign convention of the $f_{3}$ and $g_{3}$ form factors is opposite in comparison with that of Ref. [9, 16], in our convention both $g_{1}$ and $g_{3}$ form factors are positively defined for neutron beta decay. In addition, our adopted normalization of $1 /\left(M_{B}+M_{b}\right)$, instead of $1 / M_{B}$ that adopted in experiments, is theoretically preferable for considering the time-reversal symmetry on the matrix elements [59].

For convenience in later discussion, we consider the scalar form factor $f_{S}\left(q^{2}\right)$ for the vector-current form factors given in Eq. (2):

$$
f_{S}^{B \rightarrow b}\left(q^{2}\right) \equiv f_{1}^{B \rightarrow b}\left(q^{2}\right)+\frac{q^{2}}{M_{B}^{2}-M_{b}^{2}} f_{3}^{B \rightarrow b}\left(q^{2}\right),
$$


which can be defined through the matrix element of the divergence of the vector current as $\left\langle b\left(p^{\prime}\right)\left|\partial_{\alpha} V_{\alpha}(0)\right| B(p)\right\rangle=$ $\left(M_{b}-M_{B}\right) f_{S}\left(q^{2}\right) \bar{u}_{b}\left(p^{\prime}\right) u_{B}(p)$ (see Appendix A for details), and also introduce a particular linear combination of the axial-vector-current form factors given in Eq. (3) as

$$
\tilde{g}_{1}^{B \rightarrow b}\left(q^{2}\right) \equiv g_{1}^{B \rightarrow b}\left(q^{2}\right)-\frac{M_{B}-M_{b}}{M_{B}+M_{b}} g_{2}^{B \rightarrow b}\left(q^{2}\right),
$$

which is defined in an alternative parametrization of $\mathcal{O}_{\alpha}^{A}(q)$ (see Appendix B for details). Both $f_{S}$ and $\tilde{g}_{1}$ form factors are relevant in lattice calculations [13].

In the literature, the vector and the axial-vector form factors at zero momentum transfer are called the vector coupling $g_{V}=f_{1}(0)$ and the axial-vector coupling $g_{A}=g_{1}(0)$, respectively. According to Weinberg's classification [17], the terms $f_{3}$ and $g_{2}$ are known as the second-class form factors, which are identically zero in the certain symmetric limit (iso-spin symmetry, $U$-spin symmetry or $V$-spin symmetry as $S U(2)$ subgroups of the flavor $S U(3)$ symmetry) within the standard model. For an example, the second-class form factors in $\Delta S=0$ decays such as neutron beta decay are prohibited from having nonzero values because of $G$-parity conservation in the iso-spin symmetry limit $\left(m_{u}=m_{d}\right)[9]$. For $\Delta S=1$ decays, the $V$-spin symmetry $\left(m_{d}=m_{s}\right)$ plays a similar role instead of the iso-spin symmetry. Observation of nonzero second-class form factors corresponds to the direct signal of flavor $S U(3)$-breaking effects in the hyperon beta decays. On the other hand, the terms $f_{3}$ and $g_{3}$ are suppressed in the evaluation of the beta-decay transition amplitude by a factor $\left[m_{l} /\left(M_{B}+M_{b}\right)\right]^{2}$ where $m_{l}$ is the charged lepton mass. Therefore, for the decay $B \rightarrow b e \bar{\nu}_{e}$, their contributions can be safely ignored. As no accurate experiment has yet been performed on muonic hyperon decays, it is hard to access information of $f_{3}$ and $g_{3}$ form factors in present experiments.

In the exact $S U(3)$ limit, the vector couplings are simply given by $S U(3)$ Clebsch-Gordan coefficients as $f_{1}(0)=$ $f_{k l m}$, while the axial-vector couplings $g_{1}(0)$ are governed by two parameters $F$ and $D$ as $g_{1}(0)=F f_{k l m}+D d_{k l m}[4$, , 9] $\left(F=0.475(4)\right.$ and $D=0.793(5)$ are quoted in Ref. [12] for the conventional Cabibbo fit). Here, $d_{k l m}$ is the totally symmetric tensor of the $S U(3)$ group. Moreover, the conserved vector current (CVC) hypothesis becomes valid in this limit. The vector part of the weak current is a conserved current like the electromagnetic current. Thus, the value of $f_{2}(0)$ is described by $f_{2}(0)=\left(\kappa_{p}-\kappa_{n}\right) f_{k l m}-3 \kappa_{n} d_{k l m}$. where $\kappa_{p}$ and $\kappa_{n}$ represent the anomalous magnetic moments of the proton and neutron. In the case of neutron beta decay, we get $f_{1}^{n \rightarrow p}(0)=1, g_{1}^{n \rightarrow p}(0)=F+D$ and $f_{2}^{n \rightarrow p}(0)=\kappa_{p}-\kappa_{n}$, respectively. For the $\Xi^{0} \rightarrow \Sigma^{+}$decay, the exact $S U(3)$ symmetry predicts that $f_{1}^{\Xi \rightarrow \Sigma}(0), g_{1}^{\Xi \rightarrow \Sigma}(0)$ and $f_{2}^{\Xi \rightarrow \Sigma}(0)$ are identical to those of neutron beta decay.

The experimental rate of the hyperon beta decays, $B \rightarrow b l \bar{\nu}$, is given by

$$
\Gamma=\frac{G_{F}^{2}}{60 \pi^{3}}\left(M_{B}-M_{b}\right)^{5}(1-3 \delta)\left|V_{u s}\right|^{2}\left|f_{1}^{B \rightarrow b}(0)\right|^{2}\left[1+3\left|\frac{g_{1}^{B \rightarrow b}(0)}{f_{1}^{B \rightarrow b}(0)}\right|^{2}+\cdots\right],
$$

where $G_{F}$ denotes the Fermi constant. The ellipsis can be expressed in terms of a power series in the small quantity $\delta=\left(M_{B}-M_{b}\right) /\left(M_{B}+M_{b}\right)$, which is regarded as the size of flavor $S U(3)$ breaking [18]. The first linear term, which should be given by $-4 \delta\left[g_{2}(0) g_{1}(0) / f_{1}(0)^{2}\right]_{B \rightarrow b}$, is safely ignored as small as $\mathcal{O}\left(\delta^{2}\right)$ since the nonzero value of the second-class form factor $g_{2}$ should be induced at first order of the $\delta$ expansion [18]. The absolute value of $g_{1}(0) / f_{1}(0)$ can be determined by measured asymmetries such as electron-neutrino correlation [9, 18]. Therefore a theoretical estimate of $f_{1}(0)$ is primarily required for the precise determination of $\left|V_{u s}\right|$. First of all, the value of $f_{1}(0)$ should be equal to the $S U(3)$ Clebsch-Gordan coefficient up to the second order in $S U(3)$ symmetry breaking, thanks to the Ademollo-Gatto theorem [19]. As the mass splitting among octet baryons is typically of the order of 10-15\%, the expected size of the second-order corrections is a few percent level. However, either the size, or the sign of the second-order corrections are somewhat controversial among various theoretical studies at present as summarized in Table [

In the bag-model [20] and quark-model calculations [21, 22], flavor $S U(3)$-breaking effects on $f_{1}(0)$ are mainly accounted for wave-function mismatches between strange and non-strange quarks. Both models predict a small negative correction. On the other hand, the $1 / N_{c}$ expansion approach including $S U(3)$ symmetry breaking up to the second order predicts a relatively large and positive correction. Recently, the full one-loop $\mathcal{O}\left(p^{4}\right)$ calculation in heavy baryon chiral perturbation theory (HBChPT) was completed by Villadoro 24]. However, the author emphasized that the $S U(3)$ version of HBChPT does not seem to be of help for the determination of $f_{1}(0)$. This is because a slow convergence of the chiral expansion is observed. It is also pointed out that a serious convergence problem is revealed by the inclusion of spin-3/2 decuplet degrees of freedom into the framework of HBChPT. Subsequently, the complete one-loop order result has been checked in a different regularization scheme, covariant baryon chiral perturbation theory $(\mathrm{CBChPT})$ 25]. Both the size and the sign of the second order corrections are found to be different from results of HBChPT. The authors of Ref. 25] have estimated partial corrections of $\mathcal{O}\left(p^{5}\right)$ in HBChPT and then reconfirmed that the convergence behavior of $S U(3)$ baryon chiral perturbation seems to be problematic as pointed out previously in Ref. [24]. Unlike the case of $K_{l 3}$ decays, the reliability of the chiral perturbation approach is questionable for the 
hyperon decays. A model independent estimate of $f_{1}(0)$ is highly required to settle both the size, and the sign of the second order corrections on $f_{1}(0)$.

The leading correction to the axial-vector coupling $g_{1}(0)$ starts at first order in symmetry breaking, while flavor $S U$ (3)-breaking effects to $f_{1}(0)$ are suppressed in first order by the Ademollo-Gatto theorem [19]. Therefore, sizable breaking corrections, which are of the order of $10 \%$ estimated from the mass splitting in the octet baryons, are to be expected in the ratio of $g_{1}(0) / f_{1}(0)$. However, the current experimental precision is not enough to provide conclusive evidence of the violation of a two-parameter $(F$ and $D)$ fit based on the conventional Cabibbo model to ratios $g_{1}(0) / f_{1}(0)$ measured in various hyperon decays $[9]$. As mentioned earlier, the $\Xi^{0} \rightarrow \Sigma^{+}$beta decay is highly sensitive to flavor $S U(3)$ breaking since the ratio $g_{1}(0) / f_{1}(0)$ of this particular decay should be identical to that of neutron beta decay if the flavor $S U(3)$ symmetry is manifest. Therefore, flavor $S U(3)$-breaking effects may be easily exposed in the $\Xi^{0} \rightarrow \Sigma^{+}$process. Indeed, the center-of-mass correction approach [26] and the $1 / N_{c}$ expansion approach [23] predict that the $\left[g_{1}(0) / f_{1}(0)\right]_{\Xi \rightarrow \Sigma}$ is smaller than the $\left[g_{1}(0) / f_{1}(0)\right]_{n \rightarrow p}$ by $8-10 \%$ and $20-30 \%$ respectively. Such sizable breaking corrections could be distinguishable in experiment. However, the first and single experiment done by the $\mathrm{KTeV}$ collaboration at Fermilab showed no indication of flavor $S U(3)$-breaking effects on $g_{1}(0) / f_{1}(0)$, measuring $\left[g_{1}(0) / f_{1}(0)\right]_{\Xi \rightarrow \Sigma}=1.32 \pm_{0.17}^{0.21}[16]$. The $\mathrm{KTeV}$ experiment reported no evidence for a nonzero second-class form factor $g_{2}$ [16] within their experimental precision. The value of $f_{2}(0) / f_{1}(0)$ have been also measured in the $\mathrm{KTeV}$ experiment using the electron energy spectrum. Their observed value, $\left[f_{2}(0) / f_{1}(0)\right]_{\Xi \rightarrow \Sigma}=3.8 \pm 2.3$, seems to be consistent with that of neutron beta decay as $\left[f_{2}(0) / f_{1}(0)\right]_{n \rightarrow p}=\kappa_{p}-\kappa_{n}=3.706$. Needless to say, its error is too large to discriminate either the exact $S U(3)$ value or other theoretical predictions. See Table II where several theoretical predictions of the value $\left[f_{2}(0)\right]_{\Xi \rightarrow \Sigma}$ are compiled.

In this context, one would tend to conclude that the predictions of the exact $S U(3)$ symmetry limit to hold better in the case of hyperons. Indeed, it is true that the mass splitting for hyperons is rather small compared to mesons. Nevertheless, as we will show from our lattice simulations, this is indeed not the case.

TABLE I: Theoretical uncertainties of $f_{1}(0)$ for the $\Xi^{0} \rightarrow \Sigma^{+}$transition process.

\begin{tabular}{lll}
\hline \hline type of result & {$\left[f_{1}(0)\right]_{\Xi^{0} \rightarrow \Sigma^{+}}$} & Reference \\
\hline bag model & 0.97 & {$[20]$} \\
quark model & 0.987 & {$[21]$} \\
quark model & 0.976 & {$[22]$} \\
$1 / N_{c}$ expansion & $1.12 \pm 0.05$ & {$[23]$} \\
full $\mathcal{O}\left(p^{4}\right) \mathrm{HBChPT}$ & $1.009{ }^{a}$ & {$[24]$} \\
full $\mathcal{O}\left(p^{4}\right)+$ partial $\mathcal{O}\left(p^{5}\right) \mathrm{HBChPT}$ & $1.004 \pm 0.026$ & {$[25]$} \\
full $\mathcal{O}\left(p^{4}\right) \mathrm{CBChPT}$ & $0.944 \pm 0.016$ & {$[25]$} \\
\hline \hline
\end{tabular}

${ }^{a}$ The value is obtained by the iso-spin relation from that of the $\Xi^{-} \rightarrow \Sigma^{0}$ transition process.

TABLE II: Theoretical predictions of $f_{2}(0)$ for the $\Xi^{0} \rightarrow \Sigma^{+}$transition process. For evaluations, we use current values of the anomalous magnetic moments of $\Sigma$ and $\Xi$ baryons [27].

\begin{tabular}{llll}
\hline \hline type of evaluation (Ref.) & formula & {$\left[f_{2}(0)\right]_{\Xi^{0} \rightarrow \Sigma^{+}}$} & {$\left[f_{2}(0)\right]_{\Xi^{0} \rightarrow \Sigma^{+}} /\left[f_{2}(0)\right]_{n \rightarrow p}$} \\
\hline exact $S U(3)$ case & $\kappa_{p}-\kappa_{n}$ & 3.706 & 1 \\
Cabibbo model [9] & $\frac{M_{\Xi}+M_{\Sigma}}{2 M_{N}}\left(\kappa_{p}-\kappa_{n}\right)$ & 4.958 & 1.338 \\
generalized CVC & $\kappa_{\Sigma^{+}}-\kappa_{\Xi^{0}}$ & 2.708 & 0.731 \\
Sirlin's formula [28] & $\frac{M_{\Xi}+M_{\Sigma}}{2 M_{\Sigma}}\left(\kappa_{\Sigma^{+}}+\frac{1}{2} \kappa_{\Sigma^{-}}\right)-\frac{M_{\Xi}+M_{\Sigma}}{2 M_{\Xi}}\left(\kappa_{\Xi^{0}}+\frac{1}{2} \kappa_{\Xi^{-}}\right)$ & 2.475 & 0.668 \\
\hline experimental value [16] & $\mathrm{N} / \mathrm{A}$ & $3.8 \pm 2.3^{a}$ & $1.03 \pm 0.62$ \\
\hline \hline
\end{tabular}

${ }^{a}$ A factor $\left(M_{\Xi}+M_{\Sigma}\right) / M_{\Xi}$, equal to $\simeq 1.9048$ is different from definitions of the $f_{2}$ form factor adopted in Ref. [16]. 


\section{SIMULATION DETAILS}

\section{A. Lattice set-up}

We have performed a quenched lattice calculation on a $L^{3} \times T=16^{3} \times 32$ lattice with a renormalization group improved gauge action, DBW2 (doubly blocked Wilson in two-dimensional parameter space) gauge action [29, 30] at $\beta=6 / g^{2}=0.87$. The inverse of lattice spacing is about $1.3 \mathrm{GeV}$, set by the $\rho$-meson mass [31], yielding a physical volume of $(2.4 \mathrm{fm})^{3}$. The spatial size $2.4 \mathrm{fm}$ is large enough to accurately calculate the axial-vector coupling $g_{A}=g_{1}(0)$ [15], which is one of the most sensitive observable to finite volume effects [14, 32, 33].

The previous quenched DWF studies by the RBC Collaboration reported that the residual chiral symmetry breaking of DWFs is significantly improved with a moderate size of the fifth-dimension. The residual quark mass for $L_{s}=16$ is measured as small as $m_{\text {res }} \sim 5 \times 10^{-4}$ in lattice units [31]. Although we work with relatively coarse lattice spacing, $a \approx 0.15 \mathrm{fm}$, good scaling behaviors of the light hadron spectrum [31], the kaon B-parameter $B_{K}[34$ and proton decay matrix elements 35 ] are observed between at $\beta=0.87(a \approx 0.15 \mathrm{fm})$ and $1.04(a \approx 0.10 \mathrm{fm})$. Therefore, we may deduce that no large scaling violation is ensured for other observables as well in our DWF calculations. In Table III some basic physics results are compiled from Ref. [31].

In this study, DWF quark propagators were generated with three lighter quark masses $m_{u d}=0.04,0.05$ and 0.06 for up and down quarks [60] and with two heavier quark masses $m_{s}=0.08$ and 0.10 for the strange quark with $L_{s}=16$ and $M_{5}=1.8$. We then take 5 different combinations between the up (down) quark and the strange quark as $\left(m_{u d}, m_{s}\right)=(0.04,0.08),(0.05,0.08),(0.06,0.08),(0.04,0.10)$ and $(0.05,0.10)$, which yield different $S U(3)$-breaking patterns characterized by $\delta=\left(M_{B}-M_{b}\right) /\left(M_{B}+M_{b}\right)$ for the $B \rightarrow b$ process in the range of 0.009 to 0.028 . Our results are analyzed on 377 configurations. Preliminary results were first reported in Ref. 36] 61].

As mentioned earlier, the previous study of neutron beta decay with the same simulation parameters successfully yields a value of $g_{1}(0) / f_{1}(0)$ as $1.212 \pm 0.027$, which just underestimates the experimental one by less than $5 \%$ [14]. This success encourages us to study flavor $S U(3)$-breaking effects in the hyperon beta decays through a comparison between neutron beta decay and $\Xi^{0}$ beta decay.

\section{B. Mass spectra and dispersion relation}

In order to compute baryon masses or beta-decay matrix elements, we use the following baryon interpolating operator

$$
\left(\eta_{X}^{S}\right)_{i j k}(t, \mathbf{p})=\sum_{\mathbf{x}} e^{-i \mathbf{p} \cdot \mathbf{x}} \varepsilon_{a b c}\left[q_{a, i}^{T}\left(\mathbf{y}_{1}, t\right) C \gamma_{5} q_{b, j}\left(\mathbf{y}_{2}, t\right)\right] q_{c, k}\left(\mathbf{y}_{3}, t\right) \times \phi\left(\mathbf{y}_{1}-\mathbf{x}\right) \phi\left(\mathbf{y}_{2}-\mathbf{x}\right) \phi\left(\mathbf{y}_{3}-\mathbf{x}\right)
$$

where $C$ is the charge conjugation matrix defined as $C=\gamma_{t} \gamma_{y}$ and the index $X \in\{B, b\}$ distinguishes between the initial $(B)$ and final $(b)$ states. The superscript $T$ denotes transpose and the indices $a b c$ and $i j k$ label color and flavor, respectively. The superscript $S$ of the interpolating operator $\eta$ specifies the smearing for the quark propagators. In this study, we use two types of source: local source as $\phi\left(\mathbf{x}_{i}-\mathbf{x}\right)=\delta\left(\mathbf{x}_{i}-\mathbf{x}\right)$ and Gaussian smeared source. Here we take $\mathbf{x}_{1}=\mathbf{x}_{2}=\mathbf{x}_{3}=\mathbf{0}$ in our calculation. As for the Gaussian smeared source, we apply the gauge-invariant Gaussian smearing [37, 38] with $N=30, \omega=4.35$. Details of our choice of smearing parameters are described in Ref. [39].

We construct two types of the two-point function for the baryon states. One interpolating operator at the source location is constructed from Gaussian smeared quark fields, while the other interpolating operator at the sink location is either constructed from local quark fields (denoted LG) or Gaussian smeared ones (denoted GG):

$$
C_{X}^{S G}\left(t-t_{\mathrm{src}}, \mathbf{p}\right)=\frac{1}{4} \operatorname{Tr}\left\{\mathcal{P}_{+}\left\langle\eta_{X}^{S}(t, \mathbf{p}) \overline{\eta_{X}^{G}}\left(t_{\mathrm{src}},-\mathbf{p}\right)\right\}\right.
$$

with $S=L$ or $G$. The projection operator $\mathcal{P}_{+}=\frac{1+\gamma_{t}}{2}$ can eliminate contributions from the opposite-parity state for $|\mathbf{p}|=0$ [40, 41]. It is rather expensive to make the Gaussian smeared interpolating operator projected onto a specific finite momentum at the source location $\left(t_{\mathrm{src}}\right)$. However, it is sufficient to project only the sink operator onto the desired momentum by virtue of momentum conservation. Thus, the quark fields at the source location are not projected onto any specific momentum in this calculation. For the momentum at the sink location $\left(t_{\text {sink }}\right)$, we take all possible permutations of the three momentum $\mathbf{p}$ including both positive and negative directions in this study.

All hadron masses are computed by using the LG-type correlators. We use the conventional interpolating operators, $\bar{u} \gamma_{5} d\left(\bar{u} \gamma_{5} s\right)$ for the $\pi(K)$ state, $\varepsilon_{a b c}\left(u_{a}^{T} C \gamma_{5} d_{b}\right) u_{c}$ for the nucleon, $\varepsilon_{a b c}\left(u_{a}^{T} C \gamma_{5} s_{b}\right) u_{c}$ for the $\Sigma$ state and $\varepsilon_{a b c}\left(s_{a}^{T} C \gamma_{5} u_{b}\right) s_{c}$ for the $\Xi$ state. All fitted values, which are obtained from the conventional single exponential fit for baryons $(N, \Sigma$ 
and $\Xi$ ) and the conventional cosh fit for mesons $(\pi$ and $K)$, are summarized in Tables $[\mathrm{V}$ and $\mathrm{V}$. Our simulated values of the pion mass range from $0.54 \mathrm{GeV}$ to $0.67 \mathrm{GeV}$.

The evaluation of the squared four-momentum transfer $q^{2}$ requires precise knowledge of the baryon energies $E_{X}(\mathbf{p})$ $(X=N, \Sigma, \Xi)$ with finite momentum. This can be achieved by an estimation of the energy with the help of the dispersion relation and the measured rest mass that can be most precisely measured. As we reported in Ref. [15], the relativistic dispersion relation

$$
E_{X}(\mathbf{p})=\sqrt{\mathbf{p}^{2}+M_{X}^{2}}
$$

where $\mathbf{p}=\left(p_{x}, p_{y}, p_{z}\right)$ with continuum-like momenta $p_{i}=\frac{2 \pi}{L} n_{i}\left(n_{i}=0,1,2, \cdots,(L-1)\right)$, is indeed fairly consistent with the energies computed at least at the four lowest nonzero momenta: $(1,0,0),(1,1,0),(1,1,1)$ and $(2,0,0)$ in our simulations. It implies that our simulations do not much suffer from large $\mathcal{O}\left(a^{2}\right)$ errors even at finite $q^{2}$. We utilize such estimated energies instead of actually measured values in our whole analysis [62].

TABLE III: The residual mass $m_{\text {res }}$, inverse lattice spacing $\left(a_{\rho}^{-1}\right.$, set by the $\rho$ meson mass), the renormalization factor of the axial-vector current $\left(Z_{A}\right)$, the pion decay constant $\left(F_{\pi}\right)$ and the Kaon decay constant $\left(F_{K}\right)$. Those values are taken from Ref. [31], where simulations are performed on a $16^{3} \times 32$ volume.

\begin{tabular}{lccccccr}
\hline \hline Gauge action $(\beta)$ & $M_{5}$ & $L_{s}$ & $m_{\text {res }}$ & $a_{\rho}^{-1}[\mathrm{GeV}]$ & $Z_{A}\left(m_{f}=-m_{\mathrm{res}}\right)$ & $F_{\pi}[\mathrm{MeV}]$ & $F_{K}[\mathrm{MeV}]$ \\
\hline DBW2 $(0.87)$ & 1.8 & 16 & $5.69(26) \times 10^{-4}$ & $1.31(4)$ & $0.77759(45)$ & $91.2(5.2)$ & $104.2(3.8)$ \\
\hline \hline
\end{tabular}

TABLE IV: Mass spectrum of non-strange hadrons (pion and nucleon) in lattice units.

\begin{tabular}{lcc}
\hline \hline$m_{u d}$ & $M_{\pi}$ & $M_{N}$ \\
\hline 0.06 & $0.5050(8)$ & $1.0821(42)$ \\
0.05 & $0.4617(9)$ & $1.0358(46)$ \\
0.04 & $0.4148(9)$ & $0.9869(50)$ \\
\hline \hline
\end{tabular}

TABLE V: Mass spectrum of strange hadrons (Kaon, $\Sigma$ and $\Xi$ ) in lattice units.

\begin{tabular}{ccccc}
\hline \hline$m_{s}$ & $m_{u d}$ & $M_{K}$ & $M_{\Sigma}$ & $M_{\Xi}$ \\
\hline 0.08 & 0.06 & $0.5455(7)$ & $1.1161(41)$ & $1.1375(39)$ \\
& 0.05 & $0.5257(8)$ & $1.0895(43)$ & $1.1210(39)$ \\
& 0.04 & $0.5055(8)$ & $1.0626(46)$ & $1.1039(40)$ \\
0.10 & 0.05 & $0.5652(8)$ & $1.1201(46)$ & $1.1741(40)$ \\
& 0.04 & $0.5462(8)$ & $1.0941(50)$ & $1.1577(41)$ \\
\hline \hline
\end{tabular}

\section{Three-point correlation functions}

We next define the finite-momentum three-point functions for the relevant components of either the local vector current $\left(\mathcal{J}_{\alpha}^{V}\right)$ or the local axial current $\left(\mathcal{J}_{\alpha}^{A}\right)$ with the interpolating operators $\eta_{B}$ and $\eta_{b}$ for the $B$ and $b$ states:

$$
\left\langle\eta_{b}\left(t^{\prime}, \mathbf{p}^{\prime}\right) \mathcal{J}_{\alpha}^{\Gamma}(t, \mathbf{q}) \bar{\eta}_{B}(0,-\mathbf{p})\right\rangle=\mathcal{G}_{\alpha}^{\Gamma, B \rightarrow b}\left(p, p^{\prime}\right) \times f\left(t, t^{\prime}, E_{B}(\mathbf{p}), E_{b}\left(\mathbf{p}^{\prime}\right)\right)+\cdots,
$$

where the initial $(B)$ and final $(b)$ states carry fixed momenta $\mathbf{p}$ and $\mathbf{p}^{\prime}$ respectively and then the current operator has a three-dimensional momentum transfer $\mathbf{q}=\mathbf{p}-\mathbf{p}^{\prime}$. Here, Dirac indices have been suppressed. The ellipsis denotes excited state contributions which can be ignored in the case of $t^{\prime}-t \gg 1$ and $t \gg 1$. The ground state contribution of the three-point correlation function is described by two parts. The first part, $\mathcal{G}_{\alpha}^{\Gamma}\left(p, p^{\prime}\right)$, is defined as

$$
\mathcal{G}_{\alpha}^{\Gamma, B \rightarrow b}\left(p, p^{\prime}\right)=\left(-i \gamma \cdot p^{\prime}+M_{b}\right) \mathcal{O}_{\alpha}^{\Gamma, B \rightarrow b}(q)\left(-i \gamma \cdot p+M_{B}\right),
$$


where $\mathcal{O}_{\alpha}^{\Gamma}(q)$ corresponds to either Eq. (2) or Eq. (3), and the factor $f\left(t, t^{\prime}, E_{B}(\mathbf{p}), E_{b}\left(\mathbf{p}^{\prime}\right)\right)$ collects all the kinematical factors, normalization of states, and time dependence of the correlation function. The trace of $\mathcal{G}_{\alpha}^{\Gamma}\left(p, p^{\prime}\right)$ with some appropriate projection operator $\mathcal{P}$ for specific combinations of $\Gamma$ and $\alpha$ yields some linear combination of form factors in each $\Gamma$ channel. On the other hand, all time dependences of the factor $f\left(t, t^{\prime}, E_{B}(\mathbf{p}), E_{b}\left(\mathbf{p}^{\prime}\right)\right)$ can be eliminated by the appropriate ratio of three- and two-point functions [42]

$$
\mathcal{R}^{B \rightarrow b}(t)=\frac{C_{\Gamma, \alpha}^{\mathcal{P}, B \rightarrow b}\left(t, \mathbf{p}^{\prime}, \mathbf{p}\right)}{C_{b}^{G G}\left(t_{\mathrm{sink}}-t_{\mathrm{src}}, \mathbf{p}^{\prime}\right)}\left[\frac{C_{B}^{L G}\left(t_{\mathrm{sink}}-t, \mathbf{p}\right) C_{b}^{G G}\left(t-t_{\mathrm{src}}, \mathbf{p}^{\prime}\right) C_{b}^{L G}\left(t_{\mathrm{sink}}-t_{\mathrm{src}}, \mathbf{p}^{\prime}\right)}{C_{b}^{L G}\left(t_{\mathrm{sink}}-t, \mathbf{p}^{\prime}\right) C_{B}^{G G}\left(t-t_{\mathrm{src}}, \mathbf{p}\right) C_{B}^{L G}\left(t_{\mathrm{sink}}-t_{\mathrm{src}}, \mathbf{p}\right)}\right]^{\frac{1}{2}},
$$

where

$$
C_{\Gamma, \alpha}^{\mathcal{P}, B \rightarrow b}\left(t, \mathbf{p}^{\prime}, \mathbf{p}\right)=\frac{1}{4} \operatorname{Tr}\left\{\mathcal{P}\left\langle\eta_{b}^{G}\left(t_{\text {sink }}, \mathbf{p}^{\prime}\right) \mathcal{J}_{\alpha}^{\Gamma}(t, \mathbf{q}) \overline{\eta_{B}^{G}}\left(t_{\mathrm{src}},-\mathbf{p}\right)\right\rangle\right\},
$$

which are calculated by the sequential source method described in Ref. [14].

In this study, we consider the hyperon decay process $B(\mathbf{p}) \rightarrow b(\mathbf{0})$ at the rest flame of the final $(b)$ state $\left(\mathbf{p}^{\prime}=\mathbf{0}\right)$, which leads to $\mathbf{q}=\mathbf{p}$. Therefore the squared four-momentum transfer is given by $q^{2}=2 M_{b}\left(E_{B}(\mathbf{p})-M_{B}\right)-\left(M_{B}-M_{b}\right)^{2}$. The energies of the initial and final baryon state is simply abbreviated as $E_{B}$ and $E_{b}$, hereafter. In this kinematics, $\mathcal{G}_{\alpha}^{\Gamma}\left(p, p^{\prime}\right)$ is represented by a simple notation $\mathcal{G}_{\alpha}^{\Gamma}(p)$. Then, the ratio (12) gives the asymptotic form as a function of the current operator insertion time $t$,

$$
\mathcal{R}^{B \rightarrow b}(t) \rightarrow \frac{1}{4} \operatorname{Tr}\left\{\mathcal{P} \mathcal{G}_{\Gamma, \alpha}^{B \rightarrow b}(q)\right\} \times \frac{1}{\sqrt{2 M_{b}^{2} E_{B}\left(E_{B}+M_{B}\right)}}
$$

in the limit when the Euclidean time separation between all operators is large, $t_{\text {sink }} \gg t \gg t_{\text {src }}$ with fixed $t_{\text {src }}$ and $t_{\text {sink }}$.

We choose particular combinations of the projection operator $\mathcal{P}$ and the current operator $\mathcal{J}_{\alpha}^{\Gamma}(\Gamma=V$ or $A)$. Two types of the projection operator, $\mathcal{P}^{t}=\mathcal{P}_{+} \gamma_{t}$ and $\mathcal{P}_{5}^{z}=\mathcal{P}_{+} \gamma_{5} \gamma_{z}$ are considered in this study. The latter operator implies that the $z$-direction is chosen as the polarized direction. We then obtain some linear combination of desired form factors from the following projected correlation functions:

$$
\begin{aligned}
& \frac{1}{4} \operatorname{Tr}\left\{\mathcal{P}^{t} \mathcal{G}_{t}^{V, B \rightarrow b}(q)\right\}=M_{b}\left(E_{B}+M_{B}\right)\left[f_{1}^{B \rightarrow b}\left(q^{2}\right)-\frac{E_{B}-M_{B}}{M_{B}+M_{b}} f_{2}^{B \rightarrow b}\left(q^{2}\right)-\frac{E_{B}-M_{b}}{M_{B}+M_{b}} f_{3}^{B \rightarrow b}\left(q^{2}\right)\right], \\
& \frac{1}{4} \operatorname{Tr}\left\{\mathcal{P}^{t} \mathcal{G}_{i}^{V, B \rightarrow b}(q)\right\}=-i q_{i} M_{b}\left[f_{1}^{B \rightarrow b}\left(q^{2}\right)-\frac{E_{B}-M_{b}}{M_{B}+M_{b}} f_{2}^{B \rightarrow b}\left(q^{2}\right)-\frac{E_{B}+M_{B}}{M_{B}+M_{b}} f_{3}^{B \rightarrow b}\left(q^{2}\right)\right], \\
& \frac{1}{4} \operatorname{Tr}\left\{\mathcal{P}_{5}^{z} \mathcal{G}_{i}^{V, B \rightarrow b}(q)\right\}=-i \varepsilon_{i j z} q_{j} M_{b}\left[f_{1}^{B \rightarrow b}\left(q^{2}\right)+f_{2}^{B \rightarrow b}\left(q^{2}\right)\right]
\end{aligned}
$$

for the vector currents $\mathcal{J}_{t}^{V}$ and $\mathcal{J}_{i}^{V}(i=x, y, z)$. Similarly, we get

$$
\begin{aligned}
\frac{1}{4} \operatorname{Tr}\left\{\mathcal{P}_{5}^{z} \mathcal{G}_{t}^{A, B \rightarrow b}(q)\right\}= & i q_{z} M_{b}\left[g_{1}^{B \rightarrow b}\left(q^{2}\right)-\frac{E_{B}+M_{B}}{M_{B}+M_{b}} g_{2}^{B \rightarrow b}\left(q^{2}\right)-\frac{E_{B}-M_{b}}{M_{B}+M_{b}} g_{3}^{B \rightarrow b}\left(q^{2}\right)\right], \\
\frac{1}{4} \operatorname{Tr}\left\{\mathcal{P}_{5}^{z} \mathcal{G}_{i}^{A, B \rightarrow b}(q)\right\}= & M_{b}\left[\left(E_{B}+M_{B}\right)\left(g_{1}^{B \rightarrow b}\left(q^{2}\right)-\frac{M_{B}-M_{b}}{M_{B}+M_{b}} g_{2}^{B \rightarrow b}\left(q^{2}\right)\right) \delta_{i z}\right. \\
& \left.-\frac{q_{i} q_{z}}{M_{B}+M_{b}}\left(g_{2}^{B \rightarrow b}\left(q^{2}\right)+g_{3}^{B \rightarrow b}\left(q^{2}\right)\right)\right]
\end{aligned}
$$

for the axial-vector currents $\mathcal{J}_{t}^{A}$ and $\mathcal{J}_{i}^{A}(i=x, y, z)$. In this calculation, we use the four nonzero three-momentum transfer $\mathbf{q}=\frac{2 \pi}{L} \mathbf{n}\left(\mathbf{n}^{2}=1,2,3,4\right)$. All possible permutations of the lattice momentum including both positive and negative directions are properly taken into account. All three-point correlation functions are calculated with a source-sink separation of 10 in lattice units, which is the same in the previous DWF calculations of the axial-vector coupling $g_{A}$ [14] and the weak matrix elements of the nucleon [15].

Here, it is worth noting that the longitudinal momentum $\left(q_{z}\right)$ dependence explicitly appears in Eq. (19) due to our choice of the polarized direction. This fact provides two kinematical constraints on determination of the three-point functions in our calculation. First, there are two types of kinematics, $q_{z} \neq 0$ and $q_{z}=0$ in the longitudinal component $(i=z)$ of Eq. (19), except for the case of $\mathbf{n}^{2}=3$ where $q_{z}$ is always nonzero. Secondly, the transverse components $(i=x$ or $y)$ of Eq. (19) are prevented from vanishing by the kinematics only if $\mathbf{n}^{2}=2$ and 3 , where two components of the momentum including the polarized direction ( $z$-direction) are nonzero. 
First of all, in Fig. 1, we plot the dimensionless projected correlators of the vector part

$$
\begin{aligned}
\Lambda_{0}^{V, B \rightarrow b} & =\frac{\frac{1}{4} \operatorname{Tr}\left\{\mathcal{P}^{t} \mathcal{G}_{t}^{V, B \rightarrow b}(q)\right\}}{M_{b}\left(E_{B}+M_{B}\right)} \\
\Lambda_{S}^{V, B \rightarrow b} & =-\frac{1}{3} \sum_{i=x, y, z} \frac{\frac{1}{4} \operatorname{Tr}\left\{\mathcal{P}^{t} \mathcal{G}_{i}^{V, B \rightarrow b}(q)\right\}}{i q_{i} M_{b}}, \\
\Lambda_{T}^{V, B \rightarrow b} & =-\frac{1}{2}\left(\frac{\frac{1}{4} \operatorname{Tr}\left\{\mathcal{P}_{5}^{z} \mathcal{G}_{x}^{V, B \rightarrow b}(q)\right\}}{i q_{y} M_{b}}-\frac{\frac{1}{4} \operatorname{Tr}\left\{\mathcal{P}_{5}^{z} \mathcal{G}_{y}^{V, B \rightarrow b}(q)\right\}}{i q_{x} M_{b}}\right)
\end{aligned}
$$

as a function of the current insertion time slice $t$ for the $\Xi^{0} \rightarrow \Sigma^{+}$process at $\left(m_{u d}, m_{s}\right)=(0.04,0.08)$ as typical examples. Good plateaus for all squared three-momentum transfer are observed in the middle region between the source and sink points. The quoted errors are estimated by a single elimination jackknife method. The lines plotted in each figure represent the average value (solid lines) and their 1 standard deviations (dashed lines) in the time-slice range $13 \leq t \leq 17$.

Similarly, we also define the dimensionless projected correlators of the axial-vector part as

$$
\begin{aligned}
\Lambda_{L}^{A, B \rightarrow b} & =\frac{\frac{1}{4} \operatorname{Tr}\left\{\mathcal{P}_{5}^{z} \mathcal{G}_{z}^{A, B \rightarrow b}(q)\right\}}{M_{b}\left(E_{B}+M_{B}\right)}, \\
\Lambda_{T}^{A, B \rightarrow b} & =-\frac{1}{2}\left(\frac{\frac{1}{4} \operatorname{Tr}\left\{\mathcal{P}_{5}^{z} \mathcal{G}_{x}^{A, B \rightarrow b}(q)\right\}}{q_{z} q_{x}}+\frac{\frac{1}{4} \operatorname{Tr}\left\{\mathcal{P}_{5}^{z} \mathcal{G}_{y}^{A, B \rightarrow b}(q)\right\}}{q_{z} q_{y}}\right), \\
\Lambda_{0}^{A, B \rightarrow b} & =\frac{\frac{1}{4} \operatorname{Tr}\left\{\mathcal{P}_{5}^{z} \mathcal{G}_{t}^{A, B \rightarrow b}(q)\right\}}{i q_{z} M_{b}},
\end{aligned}
$$

which are also evaluated from the plateau of the ratio (12). Fig. 2 is plotted for $\Lambda_{L}^{A}$, which explicitly depends on the longitudinal momentum $q_{z}$ because of the chosen direction of the polarization. Two figures represent two types of kinematics, $q_{z} \neq 0$ and $q_{z}=0$. Solid and dashed lines are defined as in Fig. 1. Good plateaus for all squared three momentum transfer $\mathbf{q}^{2}$ are observed, similar to what we observe in the vector channel.

The remaining two ratios $\Lambda_{T}^{A}$ and $\Lambda_{0}^{A}$ are shown in Fig. 3. The upper figure is for $\Lambda_{T}^{A}$, which is accessible only for $\mathbf{q}^{2}=2$ and 3 in units of $(2 \pi / L)^{2}$, where two components of the momentum including the polarized direction $(z$ direction) are allowed to be nonzero. Again, we observe reasonable good plateaus in the time-slice range $13 \leq t \leq 17$. However, in the lower figure, the ratio $\Lambda_{0}^{A}$ at two lower $\mathbf{q}^{2}$ doesn't exhibit a clear plateau, while the reasonable plateau can be observed at two higher $\mathbf{q}^{2}$ similar to other ratios. We count a short shoulder plateau in the time-slice range $13 \leq t \leq 15$ to take the average value of $\Lambda_{0}^{A}$ at two smaller $\mathbf{q}^{2}$. It is worth mentioning that $g_{1}\left(q^{2}\right)$ and $g_{3}\left(q^{2}\right)$ are mainly determined by either $\Lambda_{L}^{A}$ or $\Lambda_{T}^{A}$, since contributions of $\Lambda_{0}^{A}$ in Eqs. (49) and (51) are numerically much smaller than others. The precise determination of the second-class form factor $g_{2}\left(q^{2}\right)$ may be affected by the poor plateau observed in $\Lambda_{0}^{A}$ at two lower q. However, the subtraction procedure for the second-class form factors $f_{3}\left(q^{2}\right)$ and $g_{2}\left(q^{2}\right)$, which will be described in the proceeding section (Sec. VIB), may reduce the systematic error stemming from above mentioned issue.

\section{Renormalization}

In general, lattice operators receive finite renormalizations relative to their continuum counterparts since the exact symmetries of the continuum are usually realized only in the continuum limit $a \rightarrow 0$. Fortunately, the well-preserved chiral and flavor symmetries of DWFs [43, 44, 45] make this task much easier than in the more conventional fermions. In this study, we use the vector and axial-vector local currents, which shares a common renormalization: $Z_{V}=Z_{A}$, up to higher order discretization errors, $\mathcal{O}\left(a^{2}\right)$ [46]. Therefore, we first focus on the vector renormalization.

The vector form factors, especially in the precise determination of $f_{1}(0)$, require some independent estimation of $Z_{V}$, the renormalization of the quark bilinear vector currents,

$$
\left[\bar{q}_{f} \gamma_{\alpha} q_{f^{\prime}}\right]^{\text {ren }}=Z_{V}^{\bar{f} f^{\prime}}\left[\bar{q}_{f} \gamma_{\alpha} q_{f^{\prime}}\right]^{\text {lattice }}
$$

where a subscript $f$ denotes the flavor index. In this study, we need two vector renormalizations, $Z_{V}^{\bar{u} d}$ and $Z_{V}^{\bar{u} s}$, for neutron beta decay and its $S U(3)$ counterpart, the $\Xi^{0} \rightarrow \Sigma^{+}$transition process. The former can be evaluated by the inverse of the forward limit of the $n \rightarrow p$ vector matrix element because of $\lim _{q^{2} \rightarrow 0}\left\langle p\left|\left[\bar{u} \gamma_{0} d\right]^{\text {ren }}\right| n\right\rangle=1$ in the present 

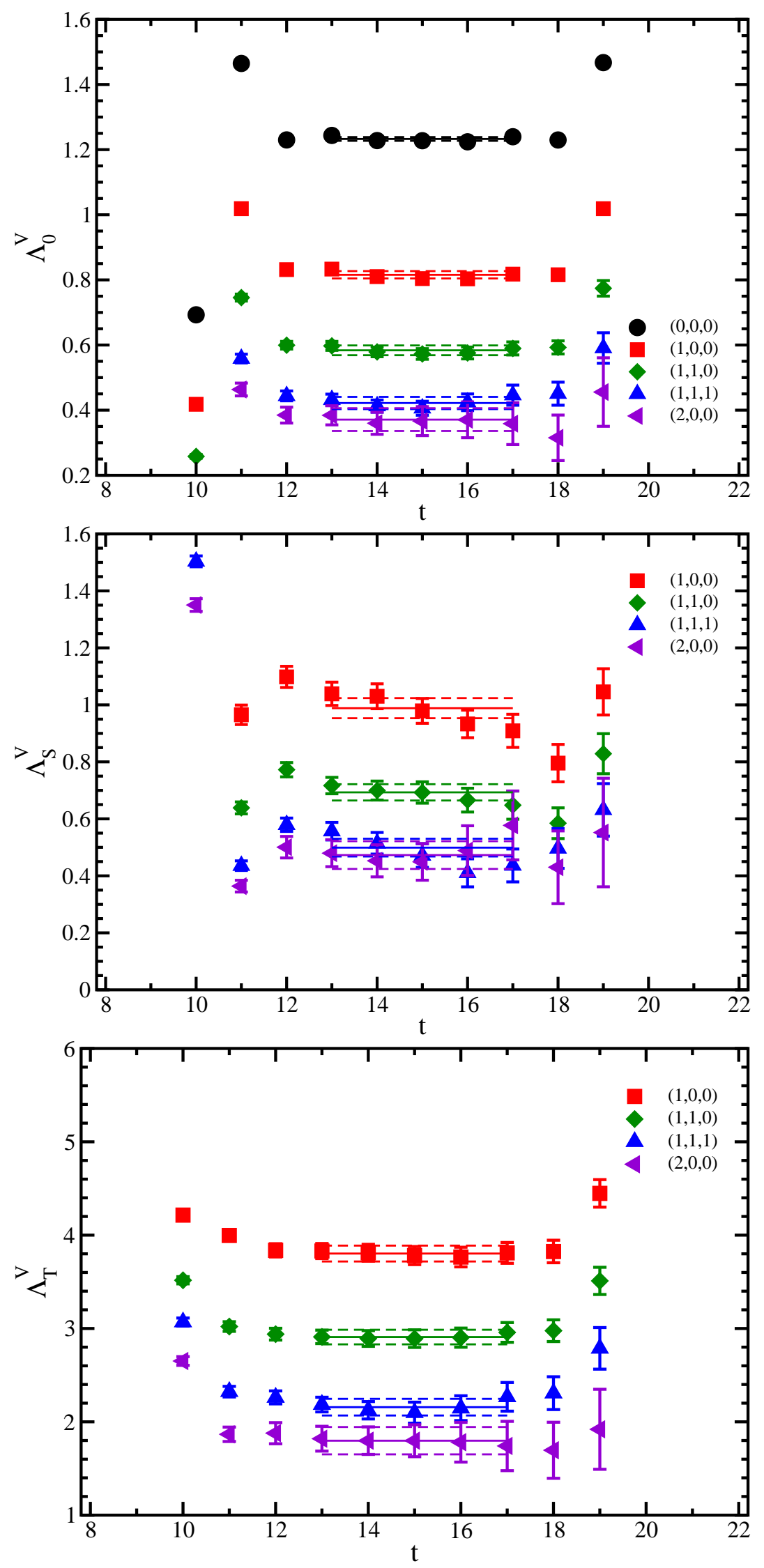

FIG. 1: Relevant ratios of three- and two-point functions, $\Lambda_{0}^{V}$ (top), $\Lambda_{S}^{V}$ (middle) and $\Lambda_{T}^{V}$ (bottom), for all possible threemomentum transfer $\mathbf{q}$ as a function of the current insertion time slice at $\left(m_{u d}, m_{s}\right)=(0.04,0.08)$. 

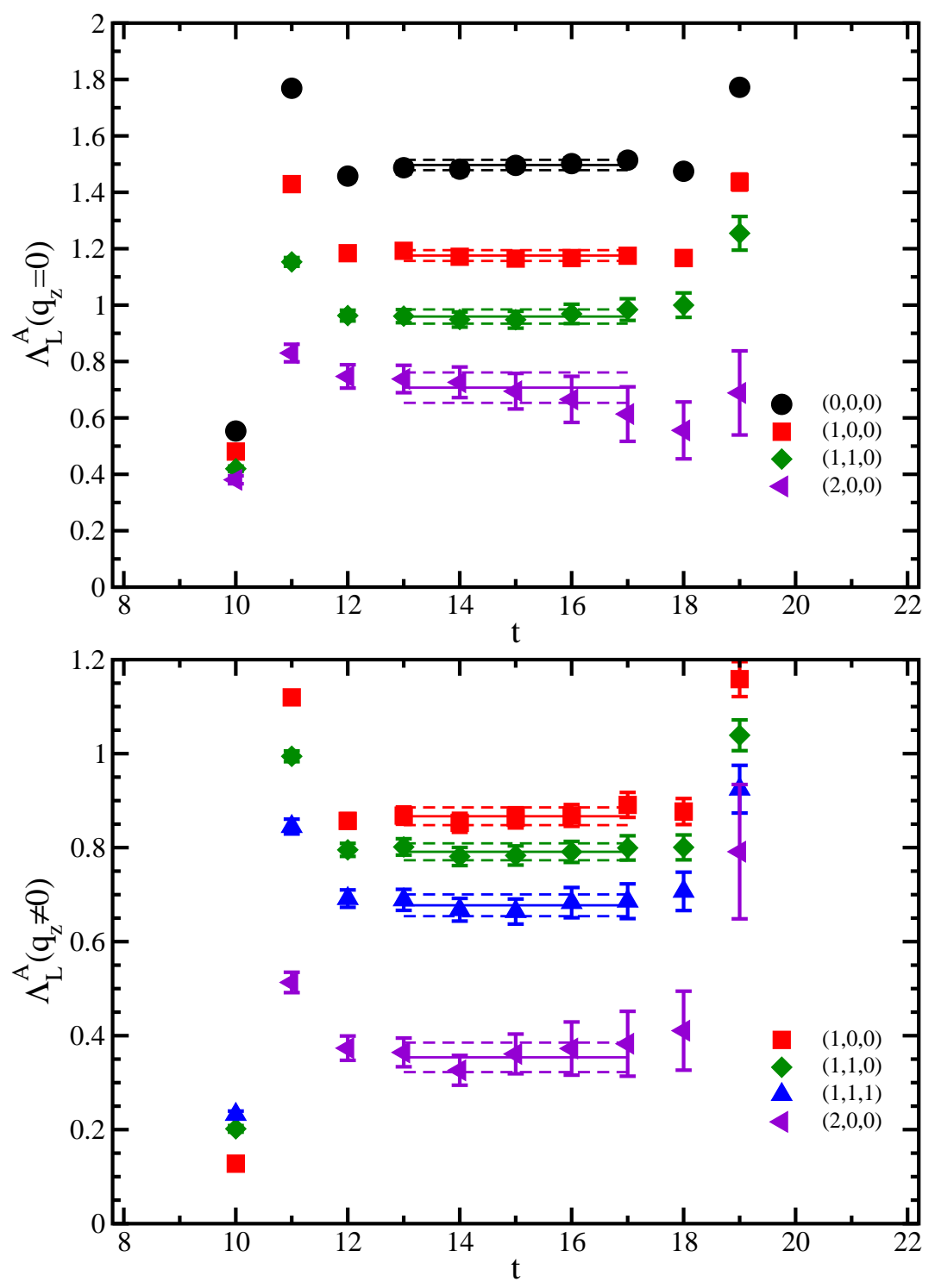

FIG. 2: Relevant ratios of three- and two-point functions, $\Lambda_{L}^{A}\left(q_{z}=0\right)$ (top) and $\Lambda_{L}^{A}\left(q_{z} \neq 0\right)$ (bottom), for all possible three-momentum transfer $\mathbf{q}$ as a function of the current insertion time slice at $\left(m_{u d}, m_{s}\right)=(0.04,0.08)$.

calculation under the exact iso-spin symmetry $\left(m_{u}=m_{d}\right)$. For the latter, this prescription is not directly applicable because of the presence of the flavor $S U(3)$ breaking. However, we may calculate $Z_{V}^{\bar{u} s}=Z_{V}^{\bar{u} s}\left(m_{u d}, m_{s}\right)$ for $m_{u d} \neq m_{s}$ through the following relation:

$$
Z_{V}^{\bar{u} s}\left(m_{l}, m_{h}\right)=\sqrt{Z_{V}^{\bar{u} s}\left(m_{l}, m_{l}\right) Z_{V}^{\bar{u} s}\left(m_{h}, m_{h}\right)}
$$

where $m_{l}$ and $m_{h}\left(m_{l}<m_{h}\right)$ are simulated quark masses for either up (down) quark or strange quark. $Z_{V}^{\bar{u} s}\left(m_{l}, m_{l}\right)$ and $Z_{V}^{\bar{u} s}\left(m_{h}, m_{h}\right)$ correspond to the case of degenerate quark masses $\left(m_{u d}=m_{s}\right)$. Therefore, for an evaluation of those two vector renormalizations, we can utilize the relation, $\lim _{q^{2} \rightarrow 0}\left\langle\Sigma^{+}\left|\left[\bar{u} \gamma_{0} s\right]^{\mathrm{ren}}\right| \Xi^{0}\right\rangle=1$, which is valid in the exact $S U(3)$ symmetry limit.

In the case of the flavor current $\left(f=f^{\prime}\right.$ in Eq.(26) $)$, we had already observed that the relation $Z_{V}=Z_{A}$ is well satisfied in the chiral limit, up to higher order discretization errors $\mathcal{O}\left(a^{2}\right)$ and neglecting explicit chiral symmetry breaking due to the moderate size of the fifth-dimensional extent $L_{s}$. This good chiral property of DWFs is known to be maintained even for the heavy-light vector and axial-vector currents [47], which correspond to the extreme case of the flavor changing current $\left(f \neq f^{\prime}\right)$ in Eq.(26). Therefore, in this study, we use the common renormalization given in Eq. (27) for both vector and axial-vector local currents. 

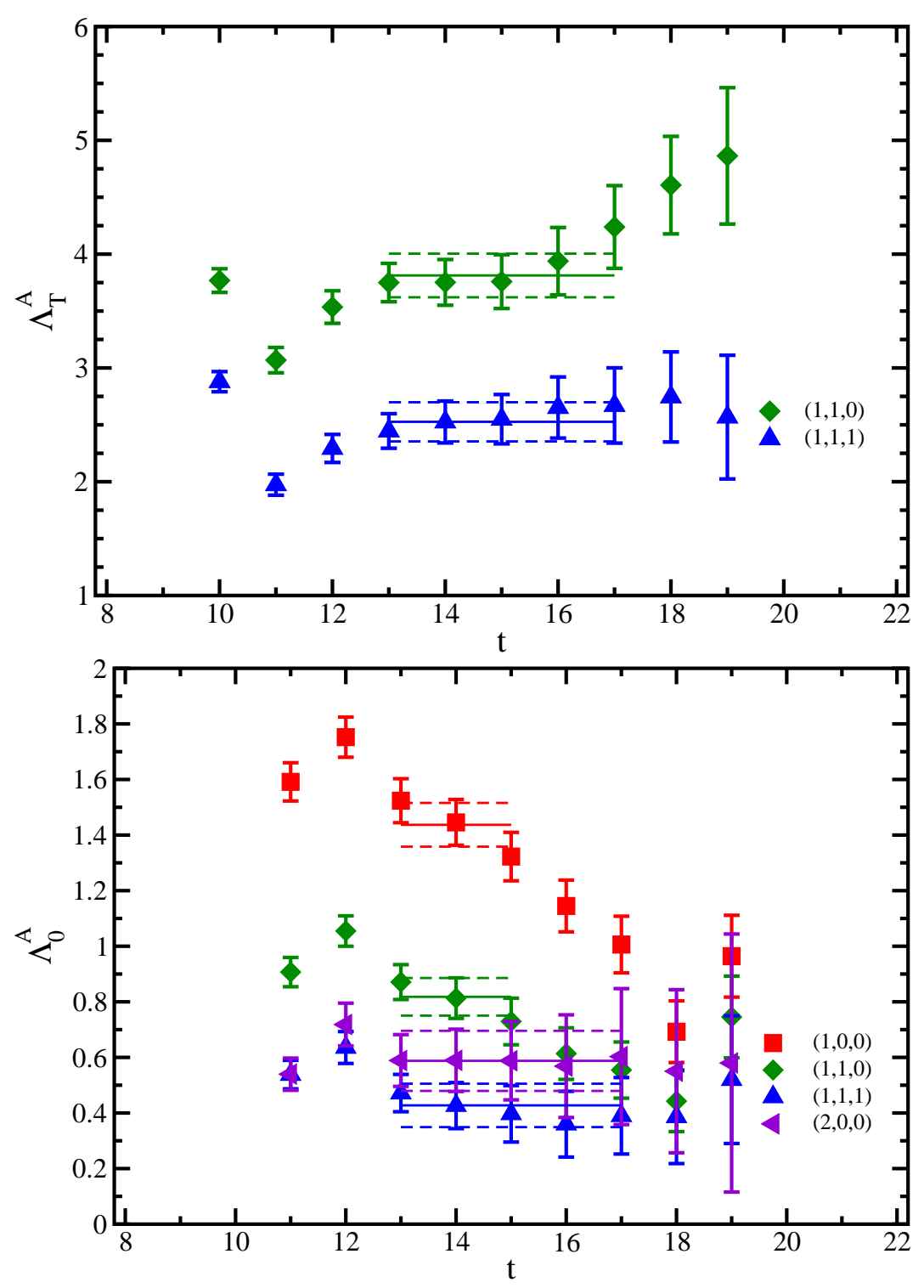

FIG. 3: Relevant ratios of three- and two-point functions, $\Lambda_{T}^{A}$ (top) and $\Lambda_{0}^{A}$ (bottom), for all possible three-momentum transfer $\mathbf{q}$ as a function of the current insertion time slice at $\left(m_{u d}, m_{s}\right)=(0.04,0.08)$.

\section{DETERMINATION OF $f_{1}(0)$}

\section{A. Scalar form factor $f_{S}\left(q^{2}\right)$ at $q^{2}=q_{\max }^{2}$}

In the vector channel, only the time component of the vector current, namely the three-point correlation function $\frac{1}{4} \operatorname{Tr}\left\{\mathcal{P}^{t} \mathcal{G}_{t}^{V, B \rightarrow b}(q)\right\}$ is prevented from vanishing at zero three momentum transfer $|\mathbf{q}|=0$ by the kinematics [14]. This non-vanishing correlator gives the scalar form factor at specific four-momentum transfer as

$$
f_{S}^{B \rightarrow b}\left(q_{\max }^{2}\right)=\Lambda_{0}^{V, B \rightarrow b}(\mathbf{q}=\mathbf{0}),
$$

where $q_{\max }^{2}=-\left(M_{B}-M_{b}\right)^{2}$ [63]. In Fig. 4, we plot the renormalized $f_{S}\left(q_{\max }^{2}\right)$ as a function of the current insertion time slice. Good plateaus are observed in the middle region between the source and sink points. The lines represent the average value (solid lines) and their 1 standard deviations (dashed lines) in the time-slice range $13 \leq t \leq 17$. We stress that the statistical accuracy is less than about $0.5 \%$ even in the worst case $\left(m_{u d}, m_{s}\right)=(0.04,0.10)$. The obtained values of the renormalized $f_{S}\left(q_{\max }^{2}\right)$ as well as the bare one and its renormalization factor $Z_{V}$ are summarized 
in Table VI. There is a tendency that the error of $f_{S}\left(q_{\max }^{2}\right)$ increases as $m_{s}$ deviates from $m_{u d}$, which was also observed in the scalar form factor of $K_{l 3}$ decays [3].

We should note that the renormalized $f_{S}\left(q_{\max }^{2}\right)$ is exactly equal to unity in the flavor $S U(3)$ symmetric limit, where $f_{S}\left(q_{\max }^{2}\right)$ becomes $f_{1}(0)$. Thus, the deviation from unity in $f_{S}\left(q_{\max }^{2}\right)$ is attributed to three types of the $S U(3)$ breaking effect: 1) the recoil correction $\left(q_{\max }^{2} \neq 0\right)$ stemming from the mass difference of $B$ and $b$ states, 2$)$ the presence of the second-class form factor $f_{3}\left(q^{2}\right)$ and 3$)$ the deviation from unity in the renormalized $f_{1}(0)$. Taking the limit of zero four-momentum transfer of $f_{S}\left(q^{2}\right)$ can separate the third effect from the others, since the scalar form factor at $q^{2}=0, f_{S}(0)$, is identical to $f_{1}(0)$. Indeed, to measure the third one is our main target.
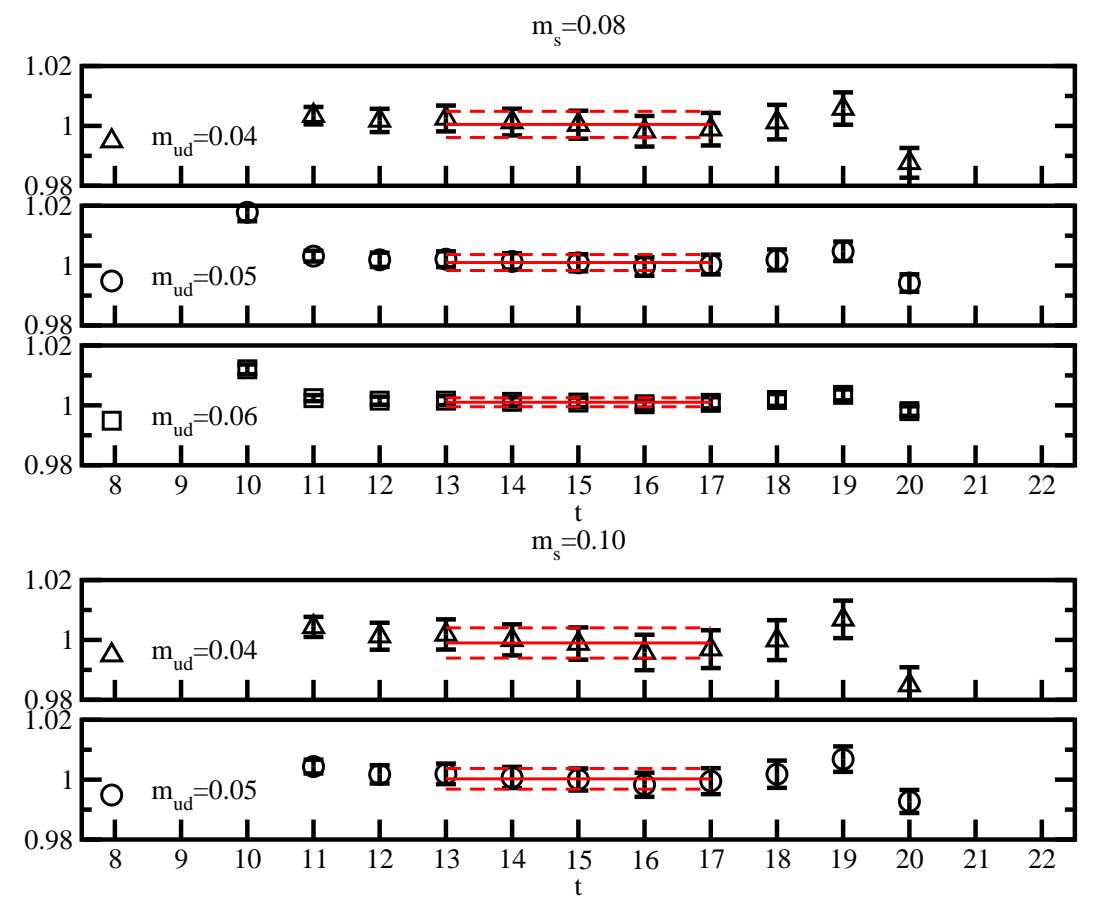

FIG. 4: $f_{S}^{\text {ren }}\left(q_{\max }^{2}\right)$ as a function of the current insertion time slice. A source-sink location of three-point functions is set at $\left[t_{\mathrm{src}}, t_{\mathrm{sink}}\right]=[10,20]$. The lines represent the average value (solid lines) and their 1 standard deviations (dashed lines) in the time-slice range $13 \leq t \leq 17$.

\section{B. Interpolation to zero four-momentum squared}

The scalar form factor $f_{S}\left(q^{2}\right)$ at $q^{2}>0$ is calculable with nonzero spatial momentum transfer $(|\mathbf{q}| \neq 0)[64]$. We can make the $q^{2}$ interpolation of $f_{S}\left(q^{2}\right)$ to $q^{2}=0$ together with the precisely measured value of $f_{S}\left(q^{2}\right)$ at $q^{2}=q_{\max }^{2}<0$. First of all, we calculate the following combinations of two projected correlators (20) and (21) with nonzero three momentum transfer $(|\mathbf{q}| \neq 0)$ :

$$
f_{S}^{B \rightarrow b}\left(q^{2}\right)=\frac{E_{B}-M_{b}}{M_{B}-M_{b}} \Lambda_{0}^{V, B \rightarrow b}-\frac{E_{B}-M_{B}}{M_{B}-M_{b}} \Lambda_{S}^{V, B \rightarrow b}
$$

and then study the $q^{2}$ dependence of the scalar form factor. In Fig. 5 , we plot the renormalized $f_{S}\left(q^{2}\right)$ as a function of four-momentum squared $q^{2}$ in physical units for $\left(m_{u d}, m_{s}\right)=(0.04,0.08)$ as a typical example. Either theoretically or phenomenologically, the $q^{2}$ dependence of $f_{S}\left(q^{2}\right)$ is not known due to the lack of knowledge of the second-class form factor $f_{3}$. Our measured $f_{S}\left(q^{2}\right)$ up to at least $q^{2}<1.0 \mathrm{GeV}^{2}$ exhibit a monotonic decrease with increasing $q^{2}$. This observation is barely consistent with an expectation that $f_{S}\left(q^{2}\right)$ is dominated by $f_{1}\left(q^{2}\right)$, which is supposed to be the dipole form at low $q^{2}$.

In practice, the lack of the precise knowledge about the $q^{2}$ dependence of $f_{S}\left(q^{2}\right)$ is not a serious issue to determine $f_{S}(0)$ reliably. The simulated value of $q_{\max }^{2}$ is not far from $q^{2}=0$ 65]. Therefore, $f_{S}(0)$ can be determined by a very short interpolation from $q_{\max }^{2}$, where we have very accurate data of $f_{S}\left(q_{\max }^{2}\right)$. This allows us to expect that the 
choice of the interpolation form does not affect the interpolated value $f_{S}(0)$ significantly. To demonstrate it, we test the monopole form

$$
f_{S}\left(q^{2}\right)=\frac{f_{S}(0)}{1+\lambda_{S}^{(1)} q^{2}}
$$

and the quadratic form

$$
f_{S}\left(q^{2}\right)=f_{S}(0)\left(1+\lambda_{S}^{(1)} q^{2}+\lambda_{S}^{(2)} q^{4}\right)
$$

for the $q^{2}$ interpolation of $f_{S}\left(q^{2}\right)$ to $q^{2}=0$. Indeed, both functional forms are adopted to evaluate $f_{S}(0)$ in a published work of the $\Sigma^{-} \rightarrow n$ transition process [13]. As shown in Fig. 囵, two determinations to evaluate $f_{S}(0)=f_{1}(0)$ from measured points are indeed consistent with each other. All obtained values of $f_{S}(0)=f_{1}(0)$ from both the monopole and quadratic form fits are summarized in Table VII. Although the quadratic fit achieves the slightly smaller value of $\chi^{2} /$ dof $\sim 0.04$ than that of the monopole fit $(\sim 0.23)$, the highest $q^{2}$ point, which is not included in our fits, rather agrees with the monopole fit. We, therefore, do not use the results from the quadratic fit in the following discussion.

Although it is hard to make a firm conclusion within the current statistical uncertainty, our measured values of $f_{1}(0)$ at the simulated points seem to receive small negative corrections of the $S U(3)$ breaking. We then introduce the parameter of flavor $S U(3)$ breaking, which is characterized by the measured mass difference between the $\Xi$ and $\Sigma$ states at the simulated points as $\delta=\left(M_{\Xi}-M_{\Sigma}\right) /\left(M_{\Xi}+M_{\Sigma}\right)$. Our observed $S U(3)$-breaking effect on $f_{1}(0)$, which corresponds to the deviation from unity, exhibits the quadratic dependence of this $S U(3)$-breaking parameter $\delta$ in consistent with the Ademollo-Gatto theorem as shown in Fig. 6. Therefore, our results indicate that a sign of the second-order correction of the $S U(3)$ breaking on $f_{1}(0)$ is likely negative.

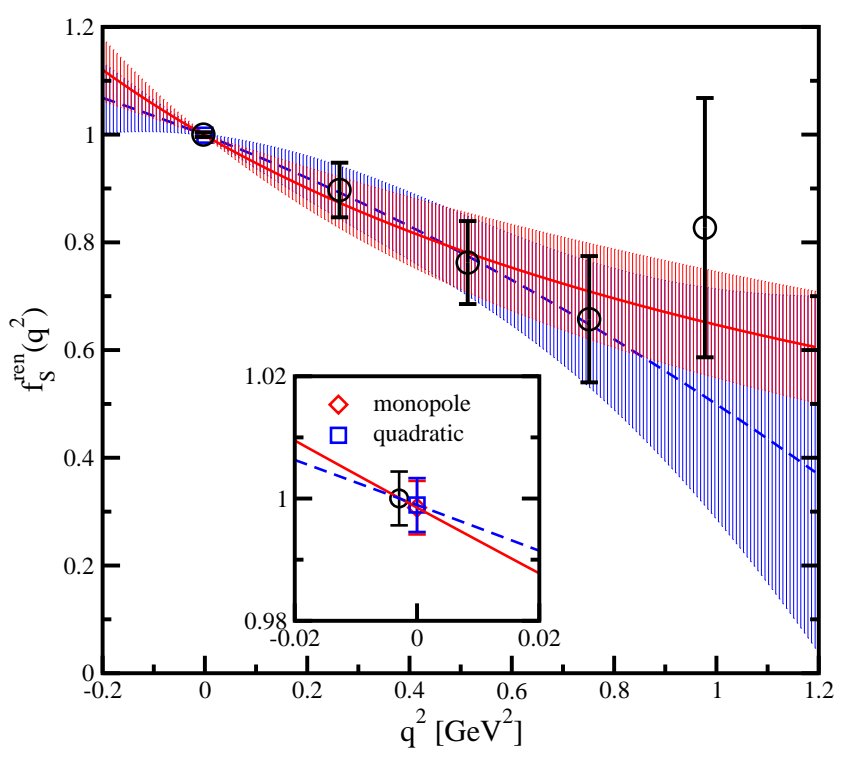

FIG. 5: Interpolation of $f_{S}\left(q^{2}\right)$ to $q^{2}=0$ for $\left(m_{u d}, m_{s}\right)=(0.04,0.08)$. Open circles are $f_{S}\left(q^{2}\right)$ at the simulated $q^{2}$. The solid (dashed) curve is the fitting result by using the monopole (quadratic) interpolation form, while the open diamond (square) represents the interpolated value to $q^{2}=0$.

\section{Chiral extrapolation of $f_{1}(0)$}

In order to estimate $f_{1}(0)$ at the physical point, we perform the chiral extrapolation of $f_{1}(0)$. As described earlier, $f_{1}(0)$ can be parameterized as

$$
f_{1}(0)=1+\Delta f
$$

where $\Delta f$ represents all $S U(3)$ breaking corrections on $f_{1}(0)$. We then introduce the following ratio:

$$
R_{\Delta f}\left(M_{K}, M_{\pi}\right)=\frac{\Delta f}{\left(M_{K}^{2}-M_{\pi}^{2}\right)^{2}},
$$




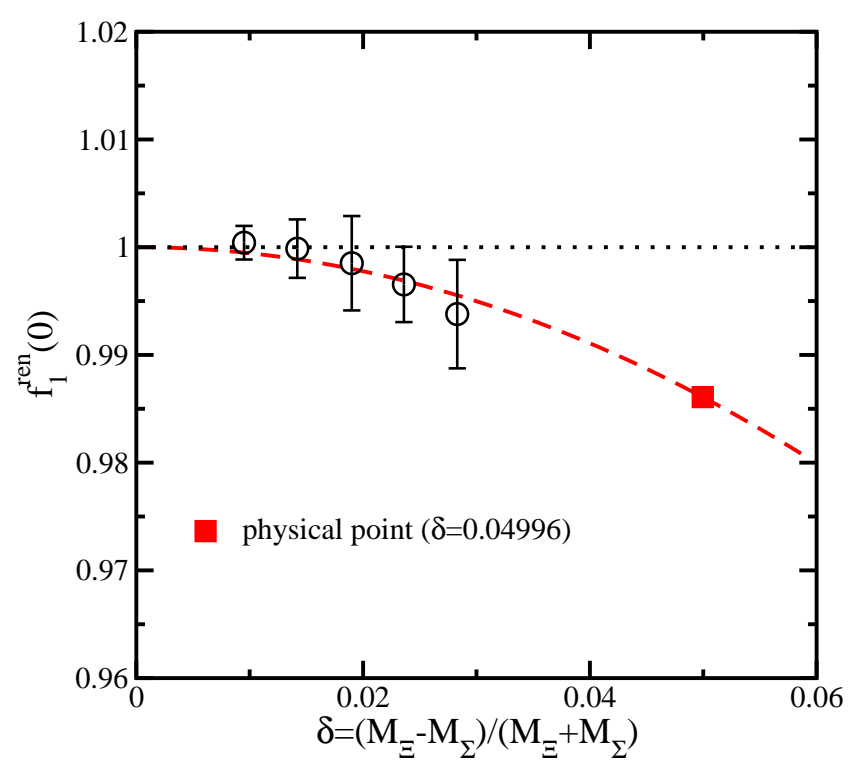

FIG. 6: The deviation from unity on $f_{1}(0)$ increases as the $S U(3)$ breaking $\delta$ increases. All measured values of $f_{1}(0)$ exhibit quadratic scaling with respect to the $S U(3)$-breaking parameter, which is suggested by the Ademollo-Gatto theorem. The dashed (quadratic) curve is a guide to the eye. The physical point is represented by a filled square.

where the leading symmetry-breaking correction, which is predicted by the Ademollo-Gatto theorem, is explicitly factorized out [13]. As reported in Ref. [13], the remaining dependence of the $S U(3)$ breaking is hardly observed within the statistical errors. To examine the quark mass dependence, we plot $R_{\Delta f}$, which is evaluated by the monopole form for the $q^{2}$ interpolation, as a function of $M_{K}^{2}+M_{\pi}^{2}$ in Fig. (7) There is no appreciable dependence of simulated quark masses within the statistical errors. This observation is consistent with what we observe in Fig, 6 where all measured values $f_{1}(0)$ at different simulated quark masses exhibit quadratic scaling with respect to the $S U(3)$-breaking parameter $\delta$. We then consider a linear fit in terms of $M_{K}^{2}+M_{\pi}^{2}$ :

$$
R_{\Delta f}\left(M_{K}, M_{\pi}\right)=A_{0}+A_{1} \cdot\left(M_{K}^{2}+M_{\pi}^{2}\right) .
$$

A dashed line in Fig. 7 corresponds to the chiral extrapolation using the linear form (34). We obtain the extrapolated value of $R_{\Delta f}$ at the physical point as

$$
R_{\Delta f}\left(M_{K}^{\text {phys }}, M_{\pi}^{\text {phys }}\right)=-0.22(24) \text { in }(\mathrm{GeV})^{-4}
$$

by employing result from the monopole form for the $q^{2}$ interpolation. We finally quote

$$
\left[f_{1}^{\mathrm{ren}}(0)\right]_{\Xi \rightarrow \Sigma}=0.987(19)
$$

at the physical point. By combining with a single estimate of $\left|V_{u s} f_{1}^{\Xi \rightarrow \Sigma}(0)\right|=0.216(33)$ from the KTeV experiment [16], we obtain

$$
\left|V_{u s}\right|=0.219(27)_{\exp }(4)_{\text {theory }},
$$

which is consistent with the value obtained from $K_{l 3}$ decays and the CKM unitary predicted value [1, 2, 3].

\section{DETERMINATION OF $g_{1}(0) / f_{1}(0)$}

\section{A. Ratio $\tilde{g}_{1}\left(q^{2}\right) / f_{S}\left(q^{2}\right)$ at $q^{2}=q_{\max }^{2}$}

Let us consider the ratio of $\Lambda_{L}^{A, B \rightarrow b}$ and $\Lambda_{0}^{V, B \rightarrow b}$, which are both accessible at zero three-momentum transfer $|\mathbf{q}|=0$ in the axial-vector and vector channels respectively. From this ratio at $|\mathbf{q}|=0$, we can evaluate the value of 


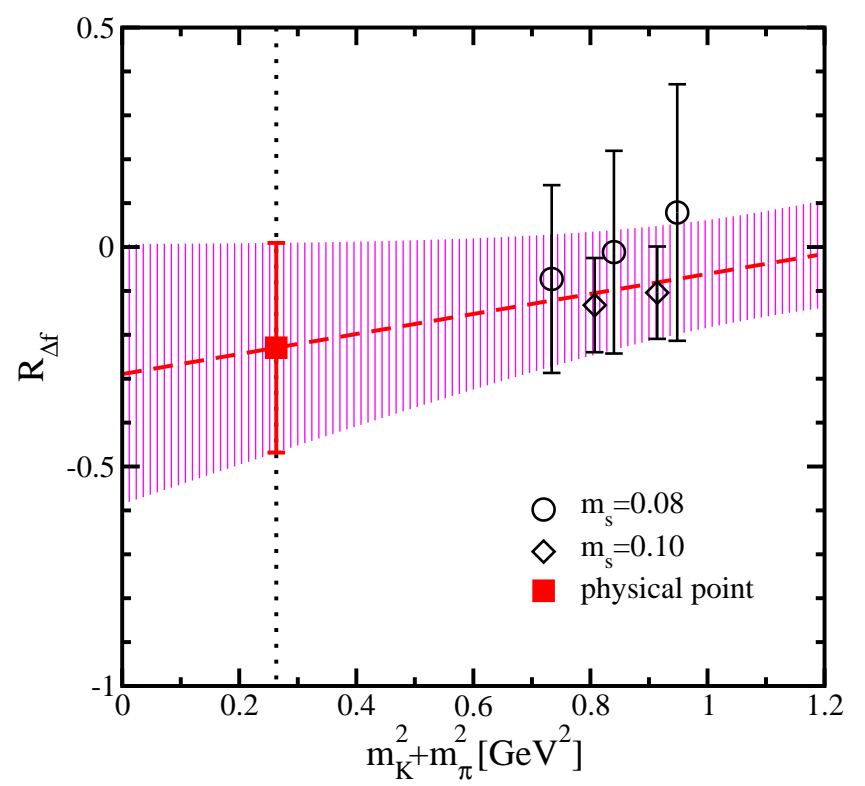

FIG. 7: Chiral extrapolation of $R_{\Delta f}$. Open circles (diamonds) are calculated with $m_{s}=0.08$ (0.10). The extrapolated point at the physical point is represented by a filled square.

TABLE VI: Results for $Z_{V}^{\text {latt }}\left(m_{u d}, m_{s}\right), f_{S}^{\text {latt }}\left(q_{\max }^{2}\right)$ and $f_{S}^{\text {ren }}\left(q_{\max }^{2}\right)$, which are evaluated in the region of $\left[t_{\min }, t_{\max }\right]=[13,17]$.

\begin{tabular}{ll|lll}
\hline \hline$m_{s}$ & $m_{u d}$ & $Z_{V}^{\text {latt }}\left(m_{u d}, m_{s}\right)$ & $f_{S}^{\text {latt }}\left(q_{\max }^{2}\right)$ & $f_{S}^{\text {ren }}\left(q_{\max }^{2}\right)$ \\
\hline 0.08 & 0.06 & $0.8144(7)$ & $1.2286(24)$ & $1.0010(15)$ \\
& 0.05 & $0.8128(8)$ & $1.2310(37)$ & $1.0010(27)$ \\
& 0.04 & $0.8112(10)$ & $1.2327(58)$ & $1.0005(44)$ \\
0.10 & 0.05 & $0.8168(7)$ & $1.2240(46)$ & $1.0003(35)$ \\
& 0.04 & $0.8152(10)$ & $1.2248(65)$ & $0.9990(51)$ \\
\hline \hline
\end{tabular}

TABLE VII: Results for $\left[f_{S}^{\text {ren }}(0)\right]_{\Xi \rightarrow \Sigma}=\left[f_{1}^{\text {ren }}(0)\right]_{\Xi \rightarrow \Sigma}$, which are evaluated by the $q^{2}$ extrapolation with the monopole and quadratic functional form.

\begin{tabular}{ll|ll}
\hline \hline & & {$\left[f_{1}^{\text {ren }}(0)\right]_{\Xi \rightarrow \Sigma}$} & \\
$m_{s}$ & $m_{u d}$ & monopole & quadratic \\
\hline 0.08 & 0.06 & $1.0004(16)$ & $1.0006(16)$ \\
& 0.05 & $0.9986(27)$ & $1.0001(28)$ \\
& 0.04 & $0.9985(44)$ & $0.9989(44)$ \\
0.10 & 0.05 & $0.9965(35)$ & $0.9972(35)$ \\
& 0.04 & $0.9938(50)$ & $0.9947(51)$ \\
\hline - phys. point & & $0.9868(191)$ & $0.9897(192)$ \\
\hline \hline
\end{tabular}

$\tilde{g}_{1}\left(q^{2}\right) / f_{S}\left(q^{2}\right)$ at $q^{2}=q_{\max }^{2}$,

$$
\frac{\tilde{g}_{1}^{B \rightarrow b}\left(q_{\max }^{2}\right)}{f_{S}^{B \rightarrow b}\left(q_{\max }^{2}\right)}=\frac{\Lambda_{L}^{A, B \rightarrow b}(\mathbf{q}=\mathbf{0})}{\Lambda_{0}^{V, B \rightarrow b}(\mathbf{q}=\mathbf{0})},
$$

where $q_{\max }^{2}=-\left(M_{B}-M_{b}\right)^{2}$. We note that this ratio is exactly equal to $g_{1}(0) / f_{1}(0)$ in the flavor $S U(3)$ symmetric limit, which corresponds to that of $n \rightarrow p$. In Fig. 8. we plot the ratio as a function of the current insertion time slice. Good plateau behaviors of $\tilde{g}_{1}\left(q_{\max }^{2}\right) / f_{S}\left(q_{\max }^{2}\right)$ are observed in the middle of region between the source and sink points. Results of $\tilde{g}_{1}\left(q_{\max }^{2}\right) / f_{S}\left(q_{\max }^{2}\right)$, which are summarized in Table VIII are averaged over appropriate time slice 
range $14 \leq t \leq 16$.

In our exploratory study [36], we proposed the following double ratio:

$$
R_{D}=\frac{\Lambda_{L}^{A, \Xi \rightarrow \Sigma}(\mathbf{q}=\mathbf{0}) \cdot \Lambda_{0}^{V, n \rightarrow p}(\mathbf{q}=\mathbf{0})}{\Lambda_{0}^{V, \Xi \rightarrow \Sigma}(\mathbf{q}=\mathbf{0}) \cdot \Lambda_{L}^{A, n \rightarrow p}(\mathbf{q}=\mathbf{0})}=\left(\frac{g_{1}^{\Xi \rightarrow \Sigma}\left(q_{\max }^{2}\right)-\delta g_{2}^{\Xi \rightarrow \Sigma}\left(q_{\max }^{2}\right)}{f_{1}^{\Xi \rightarrow \Sigma}\left(q_{\max }^{2}\right)-\delta f_{3}^{\Xi \rightarrow \Sigma}\left(q_{\max }^{2}\right)}\right) /\left(\frac{g_{1}^{n \rightarrow p}(0)}{f_{1}^{n \rightarrow p}(0)}\right),
$$

where $\delta=\frac{M_{\Xi}-M_{\Sigma}}{M_{\Xi}+M_{\Sigma}}$. Since this double ratio is exactly equal to unity in the flavor $S U(3)$ symmetric limit, the deviation form unity exposes flavor $S U(3)$-breaking effects in the $\Xi \rightarrow \Sigma$ decay. As shown in Fig. 9, the double ratio exhibits good plateau behavior slightly above unity in the time slice range $13 \leq t \leq 17$. The deviation from unity becomes large as increasing the size of the flavor $S U(3)$-breaking, which is characterized by the size of $\delta$ as listed in Table VIII

The observed deviation indeed contains three types of the $S U(3)$ breaking effect, similar to what we explained for $f_{S}\left(q_{\max }^{2}\right)$. Here, we note that $g_{1}(0) / f_{1}(0)$ receives the first-order corrections since the axial-vector form factors are not protected by the Ademollo-Gatto theorem. Therefore, we expect that the flavor $S U(3)$-breaking observed in the double ratio could be dominated by the leading symmetry-breaking correction on $\left[g_{1}(0) / f_{1}(0)\right]_{\Xi \rightarrow \Sigma}$. The reasons as follows: 1) The $q^{2}$ dependence of form factors at $q^{2}=q_{\max }^{2}$, which is proportional to $\delta^{2}$, can be involved in the second-order corrections as $f_{1}^{\Xi \rightarrow \Sigma}\left(q_{\max }^{2}\right)=f_{1}^{\Xi \rightarrow \Sigma}(0)+\mathcal{O}\left(\delta^{2}\right)$ and $g_{1}^{\Xi \rightarrow \Sigma}\left(q_{\max }^{2}\right)=g_{1}^{\Xi \rightarrow \Sigma}(0)+\mathcal{O}\left(\delta^{2}\right)$. 2) The nonzero value of the second-class form factors $f_{3}$ and $g_{2}$ starts from the first-order corrections. These contributions in $\tilde{g_{1}}$ and $f_{S}$ are involved in the second-order corrections as well. As a result, the double ratio is expressed by

$$
R_{D}=\frac{\left[g_{1}(0) / f_{1}(0)\right]_{\Xi \rightarrow \Sigma}}{\left[g_{1}(0) / f_{1}(0)\right]_{n \rightarrow p}}+\mathcal{O}\left(\delta^{2}\right)
$$

where the first term is responsible for the leading first-order correction. The deviation from unity observed in the double ratio may be able to exhibit the size of the leading $S U(3)$-breaking correction on $g_{1}(0) / f_{1}(0)$ for small $\delta$ [66]. As listed in Table VIII the observed size of flavor $S U(3)$ breaking effects is indeed comparable of the size of the leading order $\mathcal{O}(\delta)$ [36] and glows linearly with increasing the parameter $\delta$.

We simply perform the linear fit in two mass combinations $M_{K}^{2}+M_{\pi}^{2}$ and $M_{K}^{2}-M_{\pi}^{2}$ on $R_{D}$ as

$$
R_{D}=B_{0}+B_{1} \cdot\left(M_{K}^{2}+M_{\pi}^{2}\right)+B_{2} \cdot\left(M_{K}^{2}-M_{\pi}^{2}\right) .
$$

We then obtain $R_{D}=1.022(31)$ at the physical point. A sign of the observed corrections to $\left[g_{1}(0) / f_{1}(0)\right]_{\Xi \rightarrow \Sigma}$ seems to be opposite to model predictions from the center-of-mass correction approach [26] and the $1 / N_{c}$ expansion approach [23]. However we recall that the observed corrections less than a few percents are too small to justify neglect of the second-order corrections in our analysis since the natural size of the flavor $S U(3)$ breaking is around $10 \%$ [67]. To make a firm conclusion, we have to removes systematic uncertainties induced by neglecting both the recoil corrections and the presence of the second-class form factors.

TABLE VIII: Results for $\tilde{g}_{1}\left(q_{\max }^{2}\right) / f_{S}\left(q_{\max }^{2}\right)$, which are evaluated in the region of $\left[t_{\min }, t_{\max }\right]=[14,16]$. The double ration $R_{D}$ and the $S U(3)$ breaking parameter $\delta$ are also listed.

\begin{tabular}{ll|lll}
\hline \hline$m_{s}$ & $m_{u d}$ & $\tilde{g}_{1}\left(q_{\max }^{2}\right) / f_{S}\left(q_{\max }^{2}\right)$ & $R_{D}$ & $\delta=\frac{M_{\Xi}-M_{\Sigma}}{M_{\Xi}+M_{\Sigma}}$ \\
\hline 0.08 & 0.06 & $1.221(13)$ & $1.0042(28)$ & $0.0095(3)$ \\
& 0.05 & $1.219(15)$ & $1.0061(55)$ & $0.0142(5)$ \\
& 0.04 & $1.218(17)$ & $1.0099(103)$ & $0.0190(7)$ \\
0.10 & 0.05 & $1.225(13)$ & $1.0114(75)$ & $0.0236(7)$ \\
& 0.04 & $1.223(15)$ & $1.0151(126)$ & $0.0283(10)$ \\
\hline & - & $1.0217(309)$ & 0.04996 \\
\hline \hline
\end{tabular}

\section{B. Results for $\tilde{g}_{1}(0) / f_{1}(0)$ and $g_{1}(0) / f_{1}(0)$}

The recoil corrections are removed by taking the limit of considered form factors to $q^{2}=0$. In similar to the case of $f_{S}\left(q^{2}\right)$, the value of $\tilde{g}_{1}(0) / f_{S}(0)$ can be evaluated by the $q^{2}$ interpolation of $\tilde{g}_{1}\left(q^{2}\right) / f_{S}\left(q^{2}\right)$ to $q^{2}=0$. The form factor $\tilde{g}_{1}^{B \rightarrow b}\left(q^{2}\right)$ at $q^{2}>0$ can be calculated by the projected correlator (23) with zero longitudinal momentum $\left(q_{z}=0\right)$ but nonzero transverse momentum $\left(q_{x}\right.$ or $\left.q_{y} \neq 0\right)[68]$ :

$$
\tilde{g}_{1}^{B \rightarrow b}\left(q^{2}\right)=\Lambda_{L}^{A, B \rightarrow b}\left(q_{z}=0\right) .
$$



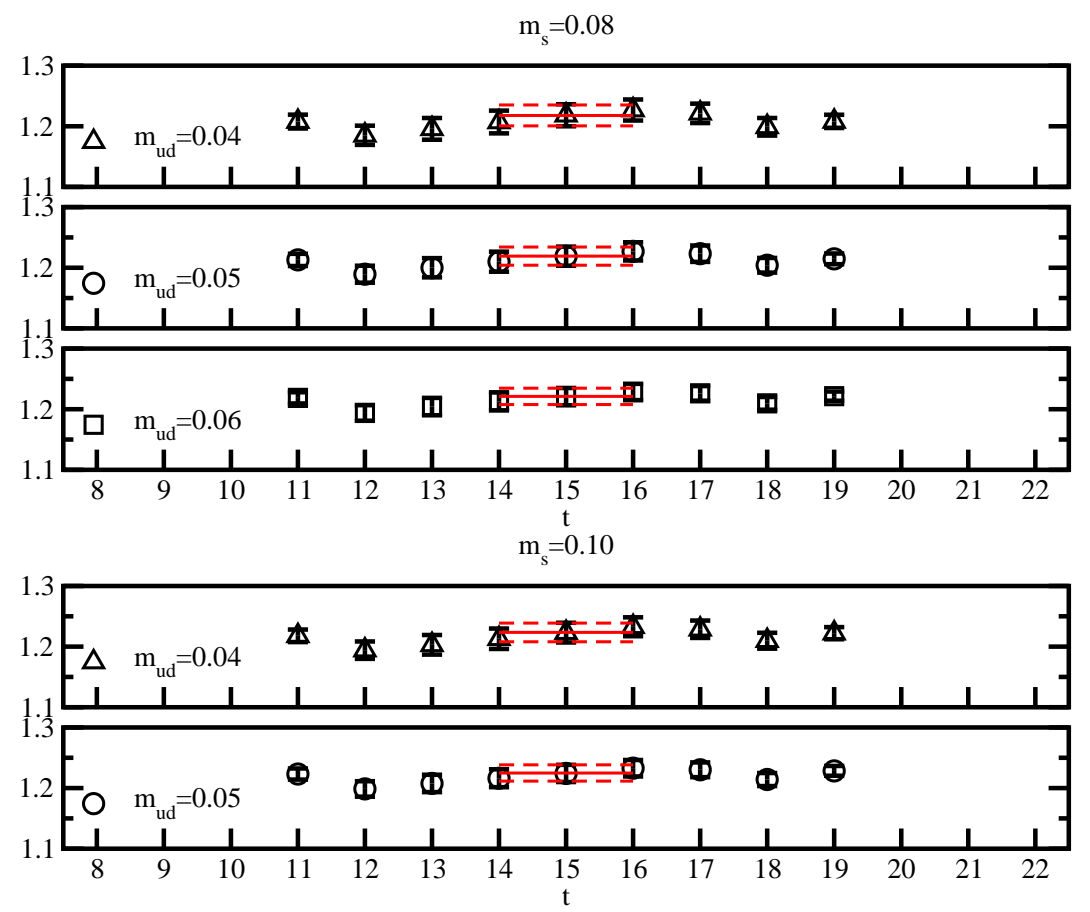

FIG. 8: $\tilde{g}_{1}\left(q_{\max }^{2}\right) / f_{S}^{\mathrm{ren}}\left(q_{\max }^{2}\right)$ as a function of the current insertion time slice. The lines represent the average value (solid lines) and their 1 standard deviations (dashed lines) in the time-slice range $14 \leq t \leq 16$.
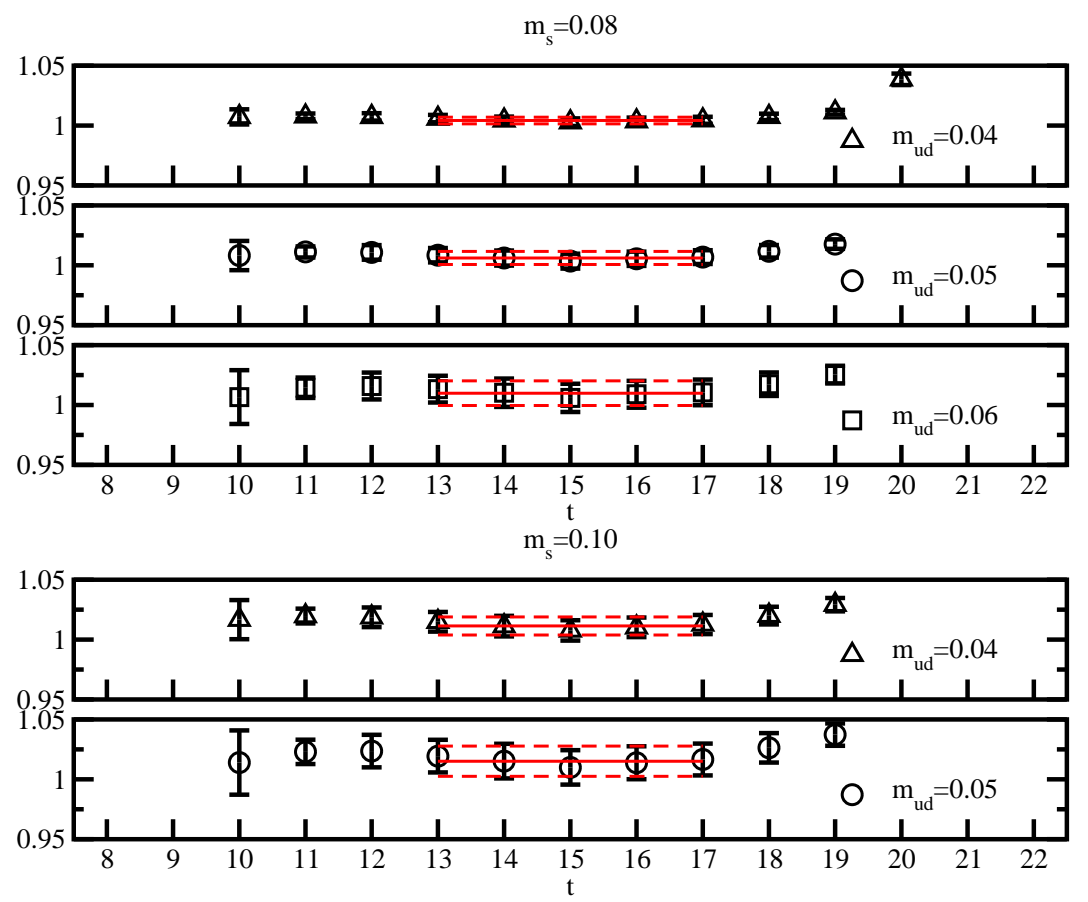

FIG. 9: The double ratio $R_{D}$ as a function of the current insertion time slice. A source-sink location of three-point functions is set at $\left[t_{\mathrm{src}}, t_{\mathrm{sink}}\right]=[10,20]$. The lines represent the average value (solid lines) and their one standard deviations (dashed lines) in the time-slice range $13 \leq t \leq 17$.

One can calculate the ratio of $\tilde{g}_{1}\left(q^{2}\right) / f_{S}\left(q^{2}\right)$ with Eqs. (28) and (42). In Fig. 10, we plot the ratio of $\tilde{g}_{1}\left(q^{2}\right) / f_{S}\left(q^{2}\right)$ as a function of $q^{2}$. We consider two types of the interpolation form, the monopole and quadratic forms, the same 
TABLE IX: Results for $\tilde{g}_{1}(0) / f_{1}(0)$, which are evaluated by the $q^{2}$ extrapolation with the monopole and quadratic functional form.

\begin{tabular}{|c|c|c|c|}
\hline$m_{s}$ & $m_{u d}$ & $\begin{array}{c}\tilde{g}_{1}(0) / f_{1}(0) \\
\text { monopole }\end{array}$ & quadratic \\
\hline \multirow[t]{3}{*}{0.08} & 0.06 & $1.220(13)$ & $1.220(13)$ \\
\hline & 0.05 & $1.217(15)$ & $1.217(15)$ \\
\hline & 0.04 & $1.215(17)$ & $1.215(17)$ \\
\hline \multirow[t]{2}{*}{0.10} & 0.05 & $1.222(13)$ & $1.222(13)$ \\
\hline & 0.04 & $1.220(15)$ & $1.220(15)$ \\
\hline- & phys. point & $1.205(27)$ & $1.206(27)$ \\
\hline
\end{tabular}

as in the case of $f_{S}\left(q^{2}\right)$. The highest $q^{2}$ data point is not included in our fits. All obtained values of $\tilde{g}_{1}(0) / f_{S}(0)=$ $\tilde{g}_{1}(0) / f_{1}(0)$ from both the monopole and quadratic form fits are summarized in Table IX. Again we observe that two determinations to evaluate $\tilde{g}_{1}(0) / f_{S}(0)$ from measured points are fairly consistent with each other. Therefore, we do not use the results from the quadratic fit in the following discussion, the same in the case of $f_{S}(0)$.

Next, we perform a linear fit in two mass combinations $M_{K}^{2}+M_{\pi}^{2}$ and $M_{K}^{2}-M_{\pi}^{2}$ for the values of $\tilde{g}_{1}(0) / f_{1}(0)$ and then obtain $\left[\tilde{g}_{1}(0) / f_{1}(0)\right]_{\Xi \rightarrow \Sigma}=1.205(27)$ at the physical point. It may be compared with its $S U(3)$-symmetric value of 1.191(49), which corresponds to the chiral extrapolated value of $\left[g_{1}(0) / f_{1}(0)\right]_{n \rightarrow p}$ to the physical point by using the simple linear fitting form in terms of $M_{\pi}^{2}$. These results give $\left[\tilde{g}_{1}(0) / f_{1}(0)\right]_{\Xi \rightarrow \Sigma}=1.016(31) \times\left[g_{1}(0) / f_{1}(0)\right]_{n \rightarrow p}$.

After the $q^{2}$ dependence is taken into account, the deviation from unity is now reduced by $0.6 \%$ from the double ratio $R_{D}$. If the conventional assumption $g_{2}(0)=0$ is adopted here similar to usual experimental analyses, the flavor $S U(3)$-breaking found in $g_{1}(0) / f_{1}(0)$ tends to be tiny. Although it does not conflict with the Cabibbo theory, the following alternative interpretation is still not ruled out. It is possible that a relatively small first-order correction on $g_{1}(0)$ is accidently canceled out in the form $\tilde{g}_{1}=g_{1}-\delta g_{2}$ by an opposite contribution stemming from the large and positive value of $g_{2}(0)$ such as $g_{2}(0) / g_{1}(0) \sim 1$. As we will discuss in next section, this is indeed the case. Therefore, we have to subtract the contribution of the second-class form factor properly in order to estimate the true size of the first-order correction on $g_{1}(0) / f_{1}(0)$.

A complete analysis requires information of the $g_{2}$ form factor. The individual form factors in Eqs. (2) and (3) can be determined at finite $|\mathbf{q}|$. Then, the value of the ratio of $g_{2}\left(q^{2}\right) / g_{1}\left(q^{2}\right)$ at zero momentum transfer are obtained by an appropriate $q^{2}$ extrapolation. We finally obtain $g_{2}(0) / g_{1}(0)=0.677(177)$ at the physical point. See the next section for details.

After the subtraction of the $g_{2}(0)$ contribution, we obtain $\left[g_{1}(0) / f_{1}(0)\right]_{\Xi \rightarrow \Sigma}=1.248(29)$ at the physical point. It implies that the relatively large and positive value of $g_{2}(0) / g_{1}(0)$ has induced a shift of the value of $\tilde{g}_{1}(0) / f_{1}(0)$ toward the exact $S U(3)$-symmetric value. Finally, we obtain

$$
\left[\frac{g_{1}(0)}{f_{1}(0)}\right]_{\Xi \rightarrow \Sigma}=1.051(35) \times\left[\frac{g_{1}(0)}{f_{1}(0)}\right]_{n \rightarrow p}
$$

at the physical point. The deviation from unity is increased by $3.5 \%$ in comparison with the unsubtracted case. Although the size of the $S U(3)$-breaking corrections on $g_{1}(0) / f_{1}(0)$ is still relatively smaller than the expected size evaluated from the mass splitting among the octet baryons $(\sim 10 \%)$, the similar size of the flavor $S U(3)$ breaking in the $\Sigma^{+} \rightarrow n$ decay was also reported in Ref. [13].

In Fig. 11, we summarize our result and the experimental values combined with predictions from the center-ofmass correction approach [26] and the $1 / N_{c}$ expansion approach [23]. Although the experimental data is not yet sufficiently precise to determine either the size, or the sign, of the $S U(3)$-breaking corrections, our result suggests that the symmetry-breaking correction is likely small but positive. It is worth mentioning that the sign of our observed corrections is opposite to the model predictions, but in agreement with that of the $\Sigma^{+} \rightarrow n$ decay measured in quenched lattice QCD [13]. 


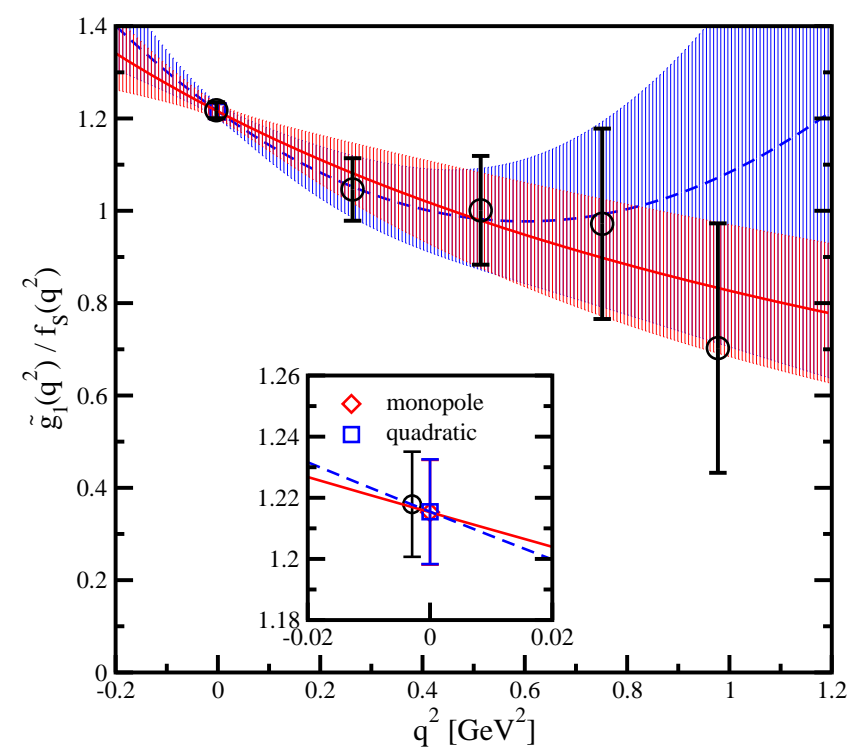

FIG. 10: Interpolation of $\tilde{g}_{1}\left(q^{2}\right) / f_{S}\left(q^{2}\right)$ to $q^{2}=0$ for $\left(m_{u d}, m_{s}\right)=(0.04,0.08)$. Open circles are $\tilde{g}_{1}\left(q^{2}\right) / f_{S}\left(q^{2}\right)$ at the simulated $q^{2}$. The solid (dashed) curve is the fitting result by using the monopole (quadratic) interpolation form, while the open diamond (square) represents the interpolated value to $q^{2}=0$.

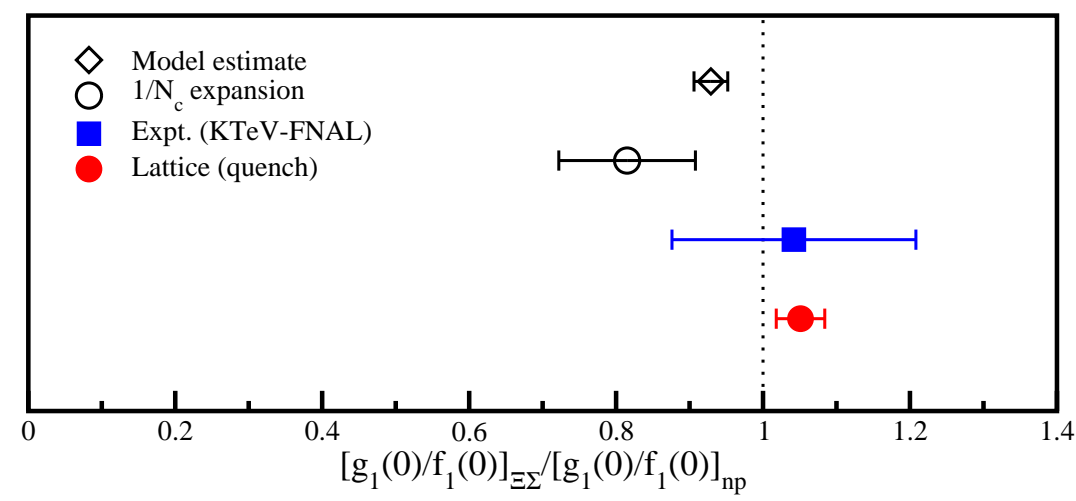

FIG. 11: Comparison among model predictions, experimental data (KTeV-FNAL) and our lattice result for the ratio of $\left[g_{1}(0) / f_{1}(0)\right]_{\Xi \rightarrow \Sigma}$ and its $S U(3)$ counterpart $\left[g_{1}(0) / f_{1}(0)\right]_{n \rightarrow p}$.

\section{RESULTS FOR OTHER FORM FACTORS}

\section{A. Computational Method}

The kinematics of $|\mathbf{q}|^{2}=0$ allows only a particular combination of the projection operator $(\mathcal{P})$ and the Lorentz index of the currents $(\alpha)$ in either vector or axial-vector channels 14]. However, in the case if spatial momentum transfer $\mathbf{q}$ is nonzero, all three-point correlation functions defined in Sec. IIIC are calculable. Therefore, three form factors at finite $|\mathbf{q}|$ can be obtained individually by solving simultaneous linear equations. For the vector channel, the simultaneous linear equations are given by

$$
\left(\begin{array}{ccc}
1 & -\frac{E_{B}-M_{B}}{M_{B}+M_{b}} & -\frac{E_{B}-M_{b}}{M_{B}+M_{b}} \\
1 & -\frac{E_{B}-M_{b}}{M_{B}+M_{b}} & -\frac{E_{B}+M_{B}}{M_{B}+M_{b}} \\
1 & 1 & 0
\end{array}\right)\left(\begin{array}{c}
f_{1}^{B \rightarrow b}\left(q^{2}\right) \\
f_{2}^{B \rightarrow b}\left(q^{2}\right) \\
f_{3}^{B \rightarrow b}\left(q^{2}\right)
\end{array}\right)=\left(\begin{array}{c}
\Lambda_{0}^{V, B \rightarrow b} \\
\Lambda_{S}^{V, B \rightarrow b} \\
\Lambda_{T}^{V, B \rightarrow b}
\end{array}\right) .
$$


One gets each form factor by inverting the above equations [69] as

$$
\begin{aligned}
f_{1}^{B \rightarrow b}\left(q^{2}\right) & =\frac{M_{B}+M_{b}}{2 M_{b}}\left[\Lambda_{0}^{V, B \rightarrow b}-\frac{E_{B}-M_{b}}{E_{B}+M_{B}} \Lambda_{S}^{V, B \rightarrow b}-\frac{M_{B}^{2}+M_{b}^{2}-2 E_{B} M_{b}}{\left(M_{B}+M_{b}\right)\left(E_{B}+M_{B}\right)} \Lambda_{T}^{V, B \rightarrow b}\right], \\
f_{2}^{B \rightarrow b}\left(q^{2}\right) & =\frac{M_{B}+M_{b}}{2 M_{b}}\left[-\Lambda_{0}^{V, B \rightarrow b}+\frac{E_{B}-M_{b}}{E_{B}+M_{B}} \Lambda_{S}^{V, B \rightarrow b}+\frac{M_{B}+M_{b}}{E_{B}+M_{B}} \Lambda_{T}^{V, B \rightarrow b}\right], \\
f_{3}^{B \rightarrow b}\left(q^{2}\right) & =\frac{M_{B}+M_{b}}{2 M_{b}}\left[\Lambda_{0}^{V, B \rightarrow b}-\frac{E_{B}+M_{b}}{E_{B}+M_{B}} \Lambda_{S}^{V, B \rightarrow b}-\frac{M_{B}-M_{b}}{E_{B}+M_{B}} \Lambda_{T}^{V, B \rightarrow b}\right] .
\end{aligned}
$$

Similarly, three-point correlation functions of the axial-vector part are also described by the following simultaneous linear equations,

$$
\left(\begin{array}{ccc}
1 & -\frac{M_{B}-M_{b}}{M_{B}+M_{b}} & 0 \\
0 & \frac{M_{b}}{M_{B}+M_{b}} & \frac{M_{b}}{M_{B}+M_{b}} \\
1 & -\frac{E_{B}+M_{B}}{M_{B}+M_{b}} & -\frac{E_{B}-M_{b}}{M_{B}+M_{b}}
\end{array}\right)\left(\begin{array}{c}
g_{1}^{B \rightarrow b}\left(q^{2}\right) \\
g_{2}^{B \rightarrow b}\left(q^{2}\right) \\
g_{3}^{B \rightarrow b}\left(q^{2}\right)
\end{array}\right)=\left(\begin{array}{c}
\Lambda_{L}^{A, B \rightarrow b}\left(q_{z}=0\right) \\
\Lambda_{T}^{A, B \rightarrow b} \\
\Lambda_{0}^{A, B \rightarrow b}
\end{array}\right)
$$

and then each individual form factor is given by inverting the above equation as

$$
\begin{aligned}
& g_{1}^{B \rightarrow b}\left(q^{2}\right)=\frac{M_{B}+M_{b}}{2 M_{b}}\left[\Lambda_{L}^{A, B \rightarrow b}\left(q_{z}=0\right)-\frac{M_{B}-M_{b}}{M_{B}+M_{b}}\left\{\Lambda_{0}^{A, B \rightarrow b}+\frac{E_{B}-M_{b}}{M_{b}} \Lambda_{T}^{A, B \rightarrow b}\right\}\right], \\
& g_{2}^{B \rightarrow b}\left(q^{2}\right)=\frac{M_{B}+M_{b}}{2 M_{b}}\left[\Lambda_{L}^{A, B \rightarrow b}\left(q_{z}=0\right)-\Lambda_{0}^{A, B \rightarrow b}-\frac{E_{B}-M_{b}}{M_{b}} \Lambda_{T}^{A, B \rightarrow b}\right], \\
& g_{3}^{B \rightarrow b}\left(q^{2}\right)=\frac{M_{B}+M_{b}}{2 M_{b}}\left[-\Lambda_{L}^{A, B \rightarrow b}\left(q_{z}=0\right)+\Lambda_{0}^{A, B \rightarrow b}+\frac{E_{B}+M_{b}}{M_{b}} \Lambda_{T}^{A, B \rightarrow b}\right] .
\end{aligned}
$$

Here we remark that as described previously, we utilize all possible permutations of the lattice momentum including both positive and negative directions and adopt four nonzero values of three-momentum transfer $\mathbf{q}=\frac{2 \pi}{L} \mathbf{n}\left(\mathbf{n}^{2}=1\right.$, $2,3,4)$. Here, it should be reminded that the $z$-direction is chosen as the polarized direction in this study. This fact makes the analysis of the axial-vector channel more complex than the vector channel. Indeed, the longitudinal momentum $\left(q_{z}\right)$ dependence explicitly enters in $\Lambda_{L}^{A}$. Accordingly, there are two types of kinematics, $q_{z} \neq 0$ and $q_{z}=0$ in the three-momentum transfer, except for the case of $\mathbf{n}^{2}=3$ where $q_{z}$ is always nonzero. In other words, $\Lambda_{L}^{A, B \rightarrow b}\left(q_{z}=0\right)$ at $\mathbf{n}^{2}=3$ can not be calculated directly. However, instead, we may evaluate $\Lambda_{L}^{A, B \rightarrow b}\left(q_{z}=0\right)$ at $\mathbf{n}^{2}=3$ from the longitudinal correlator $\Lambda_{L}^{A, B \rightarrow b}$ and the transverse correlator $\Lambda_{T}^{A, B \rightarrow b}$ by using a relation

$$
\Lambda_{L}^{A, B \rightarrow b}\left(q_{z}=0\right)=\Lambda_{L}^{A, B \rightarrow b}\left(q_{z} \neq 0\right)+\frac{q_{z}^{2}}{M_{b}\left(E_{B}+M_{B}\right)} \Lambda_{T}^{A, B \rightarrow b},
$$

which is easily read off from Eq. (19). Note that $\Lambda_{L}^{A}\left(q_{z} \neq 0\right)$ are always calculable at finite $|\mathbf{q}|$.

\section{B. Second-class form factors: $f_{3}\left(q^{2}\right)$ and $g_{2}\left(q^{2}\right)$}

\section{Subtraction method}

Figure 12 shows the ratios of $f_{3}\left(q^{2}\right) / f_{1}\left(q^{2}\right)$ (left panel) and $g_{2}\left(q^{2}\right) / g_{1}\left(q^{2}\right)$ (right panel) as a function of fourmomentum squared $q^{2}$ for $\left(m_{u d}, m_{s}\right)=(0.04,0.08)$. Open circles represent the values measured for $\Xi \rightarrow \Sigma$ at the simulated $q^{2}$. Although we observe non-negligible values of the second-class form factors, it is still questionable whether nonzero signals correspond to a pure effect from the flavor $S U(3)$ breaking. Indeed, the same analysis for the case of the exact $S U(3)$ limit $(n \rightarrow p)$, yields comparable values of the second-class form factors to those of $\Xi \rightarrow \Sigma$. Lower and upper triangle symbols correspond to the cases of $n \rightarrow p$ with $m_{u d}=0.04$ and 0.08 . The lighter $m_{u d}$ calculations yield central values closer to those of $\Xi \rightarrow \Sigma$ with larger statistical uncertainties, while results from the heavier $m_{u d}$ calculations are marginally consistent with zero values for both $f_{3}$ and $g_{2}$ form factors within 1-2 standard deviation. Although it seems that observed nonzero values of the second-class form factors suffer much from large statistical fluctuations, we are also concerned about another possibility.

For the case of the $f_{3}$ form factor, a nonvanishing contribution even in the exact $S U(3)$ limit stems from a subtle difference of $\Lambda_{S}^{V}$ and $\Lambda_{0}^{V}$ correlators, which is possibly due to the discretization error [70]. Although there is no 
such clear correspondence in the case of the $g_{2}$ form factor, the discretization error may equally cause a systematic uncertainty on the determination of the $g_{2}$ form factors as well.

To control both statistical fluctuations and systematic uncertainties, we subtract the measured values of the secondclass form factors in the $S U(3)$ limit calculation from those of $\Xi \rightarrow \Sigma$ as

$$
\begin{aligned}
& {\left[f_{3}^{\mathrm{sub}}\left(q^{2}\right)\right]_{\Xi \rightarrow \Sigma}=\left[f_{3}\left(q^{2}\right)\right]_{\Xi \rightarrow \Sigma}-\frac{M_{\Xi}+M_{\Sigma}}{2 M_{N}}\left[f_{3}\left(q^{2}\right)\right]_{n \rightarrow p},} \\
& {\left[g_{2}^{\mathrm{sub}}\left(q^{2}\right)\right]_{\Xi \rightarrow \Sigma}=\left[g_{2}\left(q^{2}\right)\right]_{\Xi \rightarrow \Sigma}-\frac{M_{\Xi}+M_{\Sigma}}{2 M_{N}}\left[g_{2}\left(q^{2}\right)\right]_{n \rightarrow p},}
\end{aligned}
$$

where a factor $\left(M_{\Xi}+M_{\Sigma}\right) /\left(2 M_{N}\right)$ is accounted for the mass difference between the $\Xi \rightarrow \Sigma$ and $n \rightarrow p$ decays, which is determined by simulated masses $[71$.

Although the $q^{2}$ value for the $\Xi \rightarrow \Sigma$ transition differs from that of the $n \rightarrow p$ transitions at the same threemomentum transfer, above subtraction is simply performed at every given three-momentum $\mathbf{q}$. There are choices to set a reference value of the subtraction, since the second-class form factors for the $n \rightarrow p$ transition are supposed to vanish with any value of $m_{u d}$. However, as mentioned above, the lighter $m_{u d}$ calculations provide larger statistical uncertainties on the second-class form factors than those of $\Xi \rightarrow \Sigma$. In Eqs. (53) and (54), we adopt the single reference values of $\left[f_{3}\left(q^{2}\right)\right]_{n \rightarrow p}$ and $\left[g_{2}\left(q^{2}\right)\right]_{n \rightarrow p}$ evaluated at $m_{u d}=0.06$. Our chosen value of $m_{u d}$ corresponds to the heaviest $m_{u d}$ mass in all combinations of $\left(m_{u d}, m_{s}\right)$ for the $\Xi \rightarrow \Sigma$ calculation in this study.

Results from the subtraction method are also plotted in Fig. 12 as filled circle symbols. It is clearly observed that statistical errors are significantly reduced after such subtraction due to a strong correlation between those two form factors, while center values are slightly shifted to zero. Non-vanishing signals of both $f_{3}$ and $g_{2}$ form factors turn out to be more pronounced. The subtraction method can expose the real $S U(3)$-breaking effect with better statistical precision.
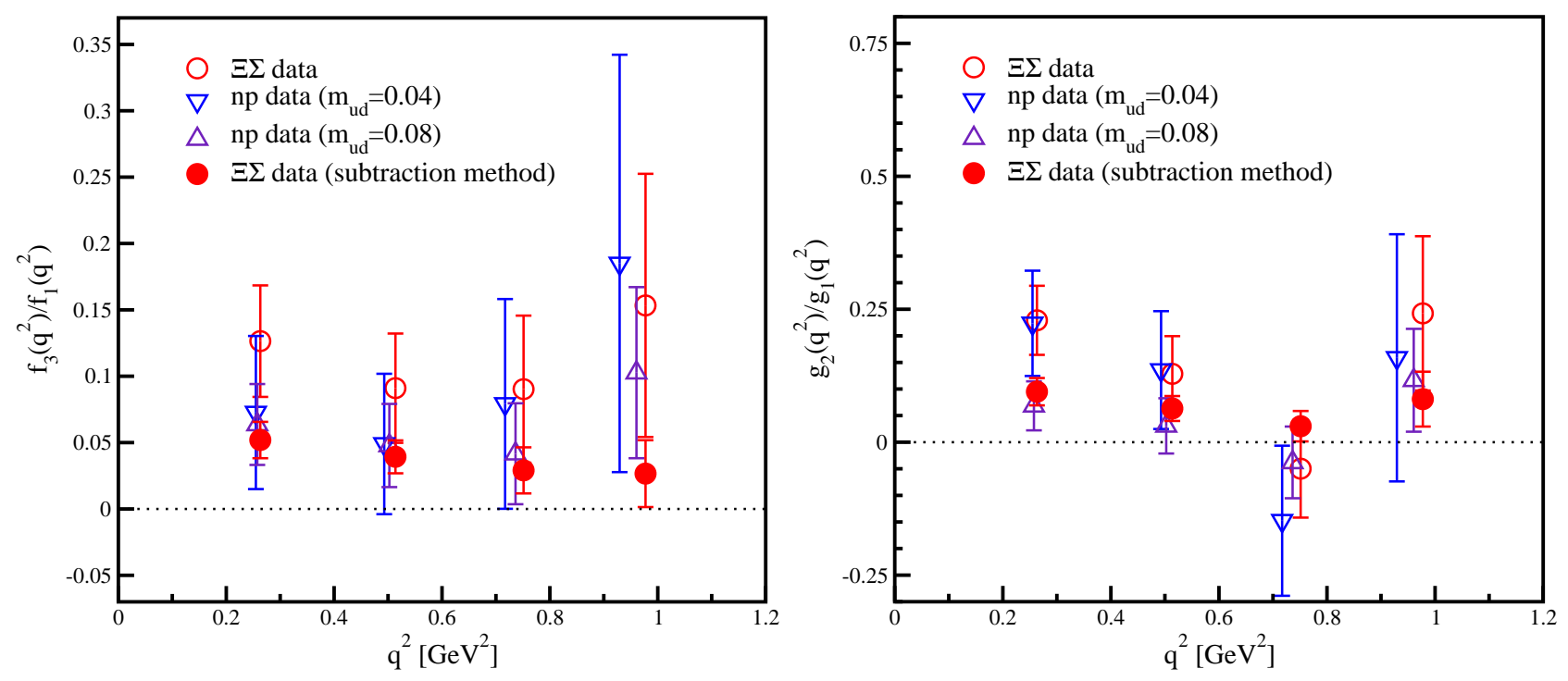

FIG. 12: The subtraction method can expose non-zero values of the second-class form factors, which corresponds to the $S U(3)$-breaking effect, with better statistical precision.

\section{Extrapolation to zero four-momentum squared}

We next examine the $q^{2}$ dependence of the ratios $f_{3}^{\text {sub }}\left(q^{2}\right) / f_{1}\left(q^{2}\right)$ and $g_{2}^{\text {sub }}\left(q^{2}\right) / g_{1}\left(q^{2}\right)$. In Fig. 13, we show the case of $\left(m_{u d}, m_{s}\right)=(0.04,0.08)$ as typical examples. One can easily observe that both ratios do not yield any strong $q^{2}$ dependence at least in our simulated range of 0.25 to $0.93 \mathrm{GeV}^{2}$. It is worth mentioning that there is no theoretical prediction of the $q^{2}$ dependence of the second-class form factors. Therefore, we simply adopt the linear or quadratic extrapolation with respect to $q^{2}$ to estimate $f_{3}(0) / f_{1}(0)$ and $g_{2}(0) / g_{1}(0)$. All evaluated values of $f_{3}(0) / f_{1}(0)$ and $g_{2}(0) / g_{1}(0)$ from two functional forms are summarized in Table $\mathrm{X}$. The extrapolated values from both determinations agree well with each other within their errors. As a result, the extrapolated values are not significantly affected by the specific fitting form adopted for describing the $q^{2}$ dependence of form factors. 

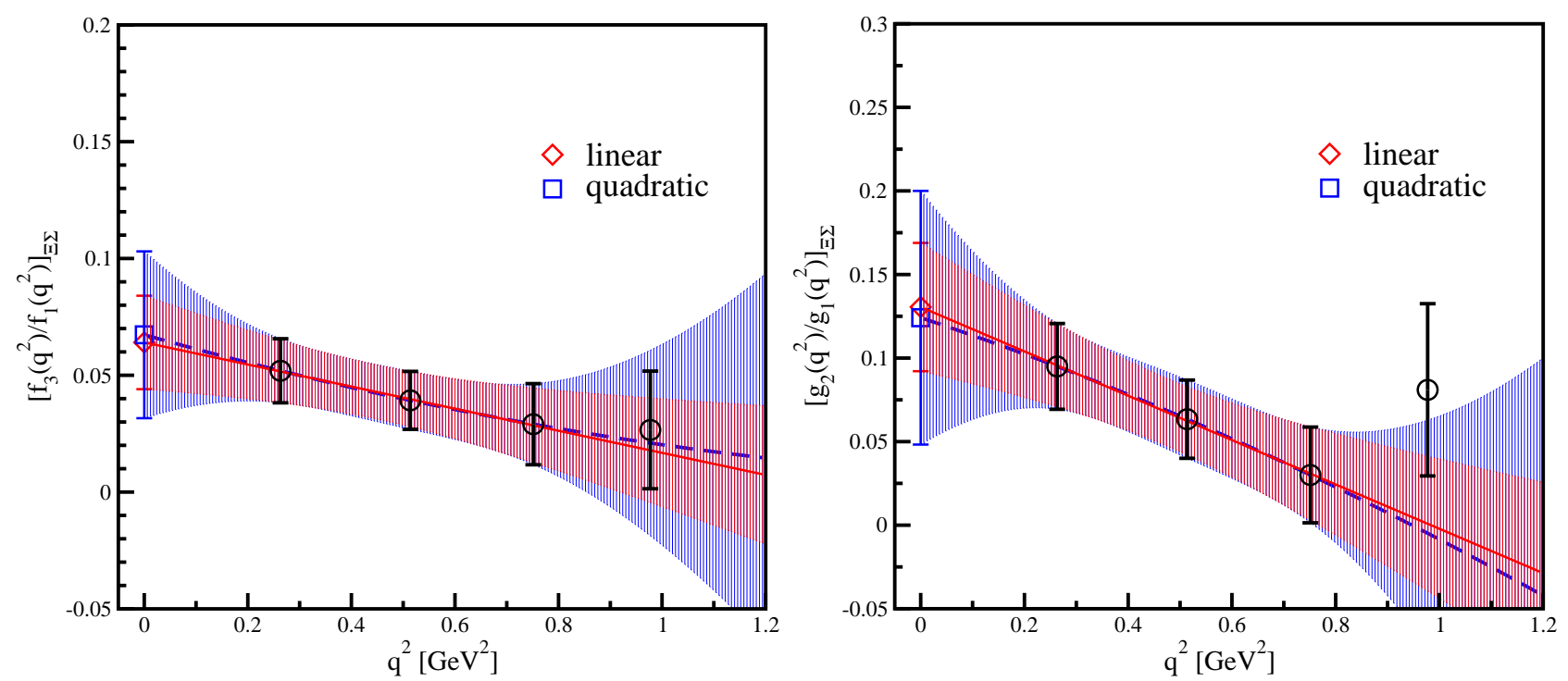

FIG. 13: The ratios $f_{3}\left(q^{2}\right) / f_{1}\left(q^{2}\right)$ (left panel) and $g_{2}\left(q^{2}\right) / g_{1}\left(q^{2}\right)$ (right panel) as functions of $q^{2}$ as in the case of $\left(m_{u d}, m_{s}\right)=$ $(0.04,0.08)$. The solid and dashed lines represent linear and quadratic fits for three lowest $q^{2}$ data, respectively.

\section{Chiral extrapolation}

In this subsection we show the results of $f_{3}(0) / f_{1}(0)$ and $g_{2}(0) / g_{1}(0)$ obtained by two types of the chiral extrapolation. In the first method, which we call method-A, we take into account the fact that the second-class form factors $f_{3}$ and $g_{2}$ vanish in the exact $S U(3)$ symmetry limit and nonzero values are induced by the first-order corrections. We then introduce the following ratios for both $f_{3}(0) / f_{1}(0)$ and $g_{2}(0) / g_{1}(0)$ :

$$
\begin{aligned}
& R_{f_{3} / f_{1}}\left(M_{K}, M_{\pi}\right)=\frac{f_{3}(0) / f_{1}(0)}{M_{K}^{2}-M_{\pi}^{2}}, \\
& R_{g_{2} / g_{1}}\left(M_{K}, M_{\pi}\right)=\frac{g_{2}(0) / g_{1}(0)}{M_{K}^{2}-M_{\pi}^{2}},
\end{aligned}
$$

where the leading first-order corrections of the flavor $S U(3)$ breaking are explicitly factorized out. In Fig. 14, we show the quark mass dependences of $R_{f_{3} / f_{1}}$ and $R_{g_{2} / g_{1}}$ as functions of $M_{K}^{2}+M_{\pi}^{2}$. The ratio $R_{f_{3} / f_{1}}$ reveals the mild dependence of $M_{K}^{2}+M_{\pi}^{2}$, while rather strong dependence of $M_{K}^{2}+M_{\pi}^{2}$ appears in the ratio $R_{g_{2} / g_{1}}$. In either cases, obtained data is well described by the linear fitting form [72]:

$$
R_{f_{3} / f_{1}}\left(M_{K}, M_{\pi}\right) \text { or } R_{g_{2} / g_{1}}\left(M_{K}, M_{\pi}\right)=A_{0}+A_{1} \cdot\left(M_{K}^{2}+M_{\pi}^{2}\right) .
$$

Here, the values of $f_{3}(0)$ and $g_{2}(0)$ given by the simplest linear fit in $q^{2}$ are used for calculating $f_{3}(0) / f_{1}(0)$ and $g_{2}(0) / g_{1}(0)$. We then get the extrapolated values of $R_{f_{3} / f_{1}}$ and $R_{g_{2} / g_{1}}$ at the physical meson masses as

$$
\begin{aligned}
& R_{f_{3} / f_{1}}\left(M_{K}^{\text {phys }}, M_{\pi}^{\text {phys }}\right)=0.61(42) \text { in }(\mathrm{GeV})^{-2}, \\
& R_{g_{2} / g_{1}}\left(M_{K}^{\text {phys }}, M_{\pi}^{\text {phys }}\right)=3.02(89) \text { in }(\mathrm{GeV})^{-2},
\end{aligned}
$$

which finally provide the values $f_{3}(0) / f_{1}(0)=0.137(94)$ and $g_{2}(0) / g_{1}(0)=0.677(177)$.

In an alternative method indicated by method-B, we may perform a linear fit in two mass combinations $M_{K}^{2}+M_{\pi}^{2}$ and $M_{K}^{2}-M_{\pi}^{2}$

$$
f_{3}(0) / f_{1}(0) \text { or } g_{2}(0) / g_{1}(0)=B_{0}+B_{1} \cdot\left(M_{K}^{2}+M_{\pi}^{2}\right)+B_{2} \cdot\left(M_{K}^{2}-M_{\pi}^{2}\right) .
$$

We then obtain $f_{3}(0) / f_{1}(0)=0.147(60)$ and $g_{2}(0) / g_{1}(0)=0.450(110)$. Both method-A and B provide consistent results. Although the errors in the latter approach are relatively smaller than that of the former, the former leads to 
a much smaller value of $\chi^{2} /$ dof than the latter. Therefore, we quote the values obtained from the method-A for our final values at the physical point:

$$
\begin{aligned}
& {\left[\frac{f_{3}(0)}{f_{1}(0)}\right]_{\Xi \rightarrow \Sigma}=0.137 \pm 0.094,} \\
& {\left[\frac{g_{2}(0)}{g_{1}(0)}\right]_{\Xi \rightarrow \Sigma}=0.677 \pm 0.177,}
\end{aligned}
$$

which show firm evidence for nonzero second-class form factors in the $\Xi^{0} \rightarrow \Sigma^{+}$beta decay. It should be reminded that the $\mathrm{KTeV}$ experiment reported no evidence for a nonzero second-class form factor $g_{2}$ [16], measuring $g_{2}(0) / f_{1}(0)=$ $-0.89 \pm 1.05$, which corresponds to $g_{2}(0) / g_{1}(0) \simeq-0.73 \pm 0.89[73]$.
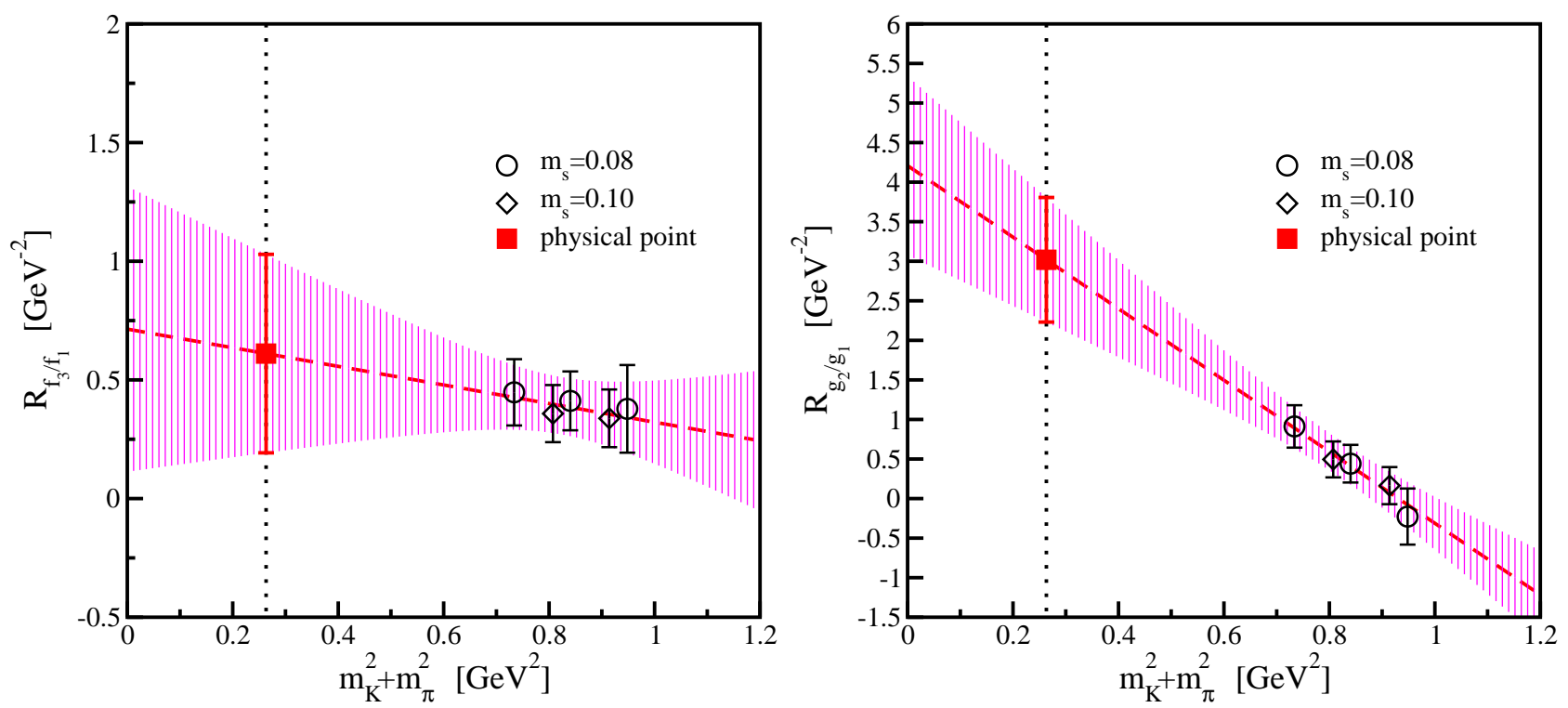

FIG. 14: Chiral extrapolation of $R_{f_{3} / f_{1}}$ (left panel) and $R_{g_{2} / g_{1}}$ (right panel). Symbols are defined as in Fig. 6.

TABLE X: Results for $\left[f_{3}(0) / f_{1}(0)\right]_{\Xi \rightarrow \Sigma}$ and $\left[g_{2}(0) / g_{1}(0)\right]_{\Xi \rightarrow \Sigma}$, both of which are evaluated by the $q^{2}$ extrapolation with the

\begin{tabular}{|c|c|c|c|c|c|}
\hline$m_{s}$ & $m_{u d}$ & $\begin{array}{l}{\left[f_{3}(0) / f_{1}(0)\right]_{\Xi \rightarrow \Sigma}} \\
\text { linear }\end{array}$ & quadratic & $\begin{array}{l}{\left[g_{2}(0) / g_{1}(0)\right]_{\Xi \rightarrow \Sigma}} \\
\text { linear }\end{array}$ & quadratic \\
\hline \multirow[t]{3}{*}{0.08} & 0.06 & $0.028(13)$ & $0.011(22)$ & $-0.017(26)$ & $0.027(52)$ \\
\hline & 0.05 & $0.045(13)$ & $0.037(22)$ & $0.048(26)$ & $0.069(49)$ \\
\hline & 0.04 & $0.064(20)$ & $0.067(36)$ & $0.131(38)$ & $0.124(76)$ \\
\hline \multirow[t]{2}{*}{0.10} & 0.05 & $0.062(22)$ & $0.040(35)$ & $0.030(43)$ & $0.088(83)$ \\
\hline & 0.04 & $0.078(26)$ & $0.066(42)$ & $0.107(49)$ & $0.143(92)$ \\
\hline (From method-A) & phys. point & $0.137(94)$ & $0.241(173)$ & $0.677(177)$ & $0.414(372)$ \\
\hline (From method-B) & phys. point & $0.147(60)$ & $0.193(110)$ & $0.450(114)$ & $0.344(232)$ \\
\hline
\end{tabular}
linear and quadratic functional form.

\section{Weak magnetism form factor $f_{2}\left(q^{2}\right)$}

In Fig. 15, we show the weak magnetism form factors $f_{2}^{B \rightarrow b}\left(q^{2}\right)$ for $\Xi \rightarrow \Sigma$ (left panel) and $n \rightarrow p$ (right panel) at $\left(m_{u d}, m_{s}\right)=(0.04,0.08)$ as functions of four-momentum squared $q^{2}$. The form factors plotted here are scaled by 
the renormalization factor $Z_{V}$ determined in Sec IV To determine $f_{2}^{\text {ren }}(0)$, we adopt two functional forms for the $q^{2}$ dependence of $f_{2}^{\text {ren }}\left(q^{2}\right)$ : the dipole form

$$
f_{2}^{\mathrm{ren}}\left(q^{2}\right)=\frac{f_{2}^{\mathrm{ren}}(0)}{\left(1+\lambda_{2}^{(1)} q^{2}\right)^{2}}
$$

and the quadratic form

$$
f_{2}^{\mathrm{ren}}\left(q^{2}\right)=f_{2}^{\mathrm{ren}}(0)\left(1+\lambda_{2}^{(1)} q^{2}+\lambda_{2}^{(2)} q^{4}\right) .
$$

The former functional form is phenomenologically adopted in the nucleon's electromagnetic form factors, which are related to the weak nucleon form factors under the CVC hypothesis on the weak processes.

Figure 15 shows that both functional forms are equally fitted to data of $f_{2}^{\text {ren }}\left(q^{2}\right)$ in either case of $\Xi \rightarrow \Sigma$ or $n \rightarrow p$ decay processes. However, there appears to be a sensitivity of the choice of the fitting form in extrapolated values at $q^{2}=0$. This is simply because our simulated $q^{2}$ points are not close enough to $q^{2}=0$. In Ref. [15], we have studied the $q^{2}$ dependence of the weak nucleon form factors at low $q^{2}$ down to about $0.1 \mathrm{GeV}^{2}$, which is accessible with the spatial extent $L=24$. Indeed, in our previous study, the weak magnetism form factor for $n \rightarrow p$ is observed to be well described by the dipole form [15]. To make this point clear, in the right panel of Fig. [15, we also include results from the larger volume $(L=24)$ for a comparison. The steep raising behavior of $L=24$ data as $q^{2}$ decreases clearly favors the dipole form. We, therefore, do not use the results from the quadratic fit in the following discussion. All extrapolated values with both determinations are summarized in Table XI. As for the $n \rightarrow p$ transition, the extrapolated values of $f_{2}(0)$ by using the dipole fit are summarized in Table XII together with other relevant quantities.

We also perform the chiral extrapolation of $f_{2}^{\text {ren }}(0)$ through a linear fit in two mass combinations $M_{K}^{2}+M_{\pi}^{2}$ and $M_{K}^{2}-M_{\pi}^{2}$, the same as Eq.(60) . We obtain $\left[f_{2}^{\text {ren }}(0)\right]_{\Xi \rightarrow \Sigma}=3.30 \pm 0.24$ by employing result from the dipole form for the $q^{2}$ extrapolation. Finally, we compare the ratio $\left[f_{2}(0) / f_{1}(0)\right]_{\Xi \rightarrow \Sigma}$ to its $S U(3)$ counterpart $\left[f_{2}(0) / f_{1}(0)\right]_{n \rightarrow p}$ and then observe an order of $16 \%$ breaking effect as

$$
\left[\frac{f_{2}(0)}{f_{1}(0)}\right]_{\Xi \rightarrow \Sigma}=1.16(11) \times\left[\frac{f_{2}(0)}{f_{1}(0)}\right]_{n \rightarrow p},
$$

which implies the violation of the exact $S U(3)$ relation. It is worth mentioning that $\left[f_{2}(0) / f_{1}(0)\right]_{\Xi \rightarrow \Sigma}$ tends to be bigger than $\left[f_{2}(0) / f_{1}(0)\right]_{n \rightarrow p}$, while the $f_{2}(0) / f_{1}(0)$ values evaluated from both the generalized CVC hypothesis [12] and Sirlin's formula 28 yield opposite results as previously shown in Table [II. In addition, the observed size of the deviation from unity in Eq. (65) may also be compared with the Cabibbo-model prediction as $\left(M_{\Xi}+M_{\Sigma}\right) /\left(2 M_{N}\right)=1.338$ [9] which corresponds to a factor accounted for the mass difference between the $\Xi \rightarrow \Sigma$ and $n \rightarrow p$ decays. We again observe a $15 \%$ deviation from the Cabibbo model.

TABLE XI: Results for renormalized $\left[f_{2}(0)\right]_{\Xi \rightarrow \Sigma}$ is evaluated by the $q^{2}$ extrapolation with the dipole and quadratic functional form.

\begin{tabular}{ll|ll}
\hline \hline & & {$\left[f_{2}^{\text {ren }}(0)\right]_{\Xi \rightarrow \Sigma}$} \\
$m_{s}$ & $m_{u d}$ & dipole & quadratic \\
\hline 0.08 & 0.06 & $3.64(11)$ & $3.25(12)$ \\
& 0.05 & $3.60(13)$ & $3.20(14)$ \\
& 0.04 & $3.54(15)$ & $3.14(16)$ \\
0.10 & 0.05 & $3.63(11)$ & $3.23(12)$ \\
& 0.04 & $3.57(13)$ & $3.17(14)$ \\
\hline - & phys. point & $3.30(24)$ & $2.92(25)$ \\
\hline \hline
\end{tabular}

\section{Induced pseudo-scalar form factor $g_{3}\left(q^{2}\right)$}

We next show the $q^{2}$ dependence of the ratio of the induced pseudo-scalar form factor $g_{3}\left(q^{2}\right)$ and the axial-vector form factor $g_{1}\left(q^{2}\right)$ at $\left(m_{u d}, m_{s}\right)=(0.04,0.08)$ in Fig. 16. It is phenomenologically known that the $q^{2}$ dependence of $g_{3}\left(q^{2}\right)$ in the low $q^{2}$ region are well fitted by the pion-pole dominance (PPD) form, $g_{3}\left(q^{2}\right)=4 M_{N}^{2} g_{1}\left(q^{2}\right) /\left(q^{2}+\right.$ $\left.M_{\pi}^{2}\right)$ [49, 50, 51]. As shown in Ref. [15], the PPD form provides a good description of the $q^{2}$ dependence of the 

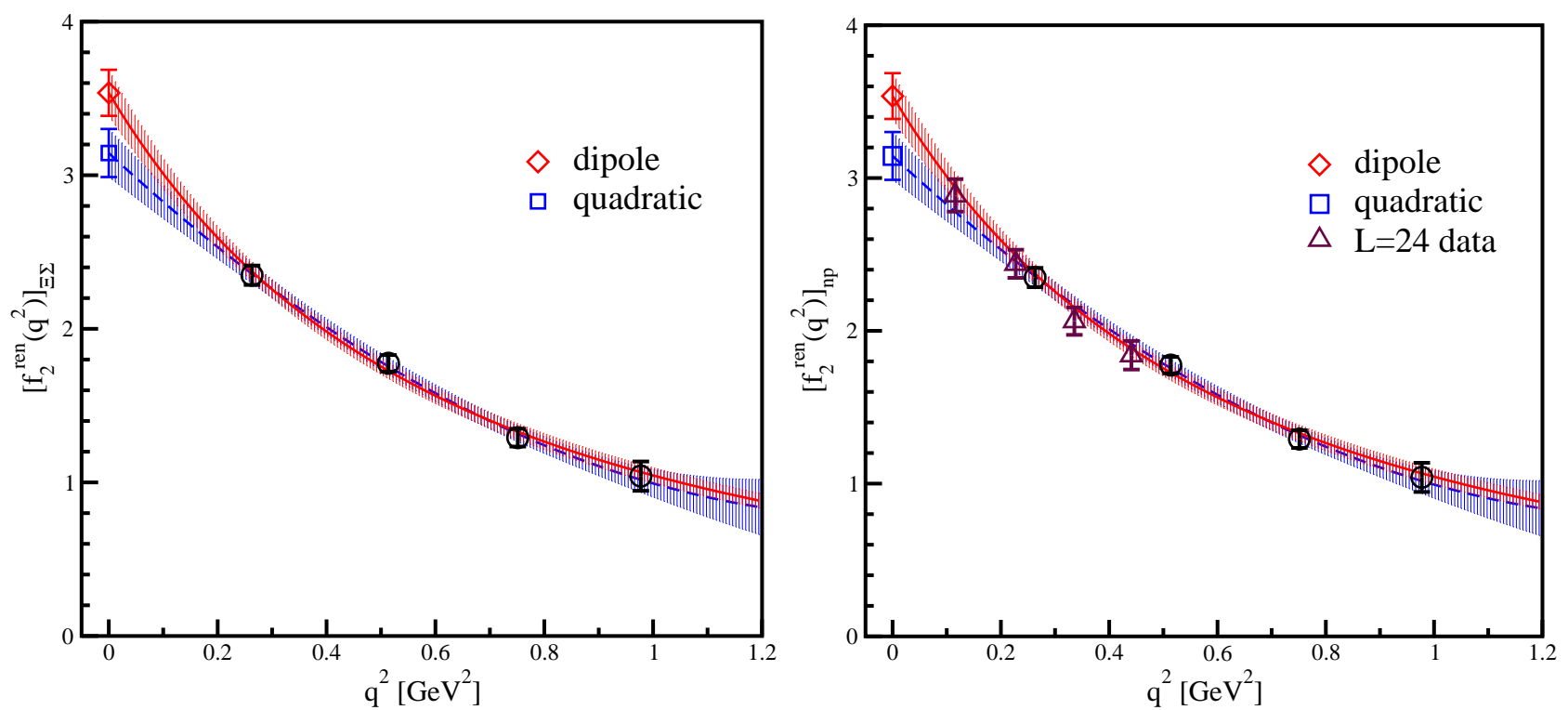

FIG. 15: The renormalized weak-magnetism form factor $f_{2}\left(q^{2}\right)$ as functions of four-momentum squared $q^{2}$ for $\Xi^{0} \rightarrow \Sigma^{+}($left panel) and $n \rightarrow p$ (right panel) at $\left(m_{u d}, m_{s}\right)=(0.04,0.08)$. For the $q^{2}$ extrapolation, we apply two types of fitting form. The solid (dashed curve) is the fitting result by using the dipole (quadratic) form, while the open diamond (square) represents the extrapolated value to $q^{2}=0$. The highest $q^{2}$ data point is not included in our fits. In the right panel, results from the larger volume $(L=24)[15]$ are also included as open triangles for a comparison.

TABLE XII: Summary for the $n \rightarrow p$ transition process. The values of $f_{2}(0) / f_{1}(0)$ determined by the dipole form for the $q^{2}$ extrapolation, while the values $g_{3}(0) / g_{1}(0)$ are evaluated by the pion-pole fit.

\begin{tabular}{c|lll}
\hline \hline$m_{u d}$ & $g_{1}(0) / f_{1}(0)$ & $f_{2}(0) / f_{1}(0)$ & $g_{3}(0) / g_{1}(0)$ \\
\hline 0.10 & $1.240(12)$ & $3.80(8)$ & $12.06(32)$ \\
0.08 & $1.226(11)$ & $3.71(10)$ & $13.52(38)$ \\
0.06 & $1.214(14)$ & $3.57(13)$ & $15.35(49)$ \\
0.05 & $1.209(18)$ & $3.47(17)$ & $16.73(59)$ \\
0.04 & $1.202(24)$ & $3.35(24)$ & $18.68(79)$ \\
\hline phys. point & $1.191(49)$ & $3.18(29)$ & $151.0(6.9)$ \\
\hline \hline
\end{tabular}

nucleon's induced pseudo-scalar form factor measured in quenched DWF simulations at least at low $q^{2}$. On the other hand, there is no direct experimental information in the case of $g_{3}\left(q^{2}\right)$ for any other hyperon decays.

Under the partial conserved axial-vector current (PCAC) hypothesis [74], an extension of PPD to $\Delta S=1$ decays predicts that the induced pseudo-scalar form factor for the hyperon beta decays, such as the $\Xi \rightarrow \Sigma$ decay, the $K$ meson pole, instead of the pion pole. In the generalized PPD form, the ratio of $g_{3}\left(q^{2}\right) / g_{1}\left(q^{2}\right)$ for the $\Xi \rightarrow \Sigma$ transition is given by a simple monopole form

$$
\left[\frac{g_{3}\left(q^{2}\right)}{g_{1}\left(q^{2}\right)}\right]_{\Xi \rightarrow \Sigma}^{\mathrm{PPD}}=\frac{\left(M_{\Xi}+M_{\Sigma}\right)^{2}}{q^{2}+M_{K}^{2}}
$$

where a monopole mass corresponds to the Kaon mass.

In Fig. 16, we include the predicted $q^{2}$ dependence (dotted curve) evaluated by the generalized PPD form with simulated baryon masses $\left(M_{N}, M_{\Sigma}, M_{\Xi}\right)$ and meson masses $\left(M_{\pi}, M_{K}\right)$. Three larger $q^{2}$ data points are quite close to predicted curves in either $\Xi \rightarrow \Sigma$ or $n \rightarrow p$, while the lowest $q^{2}$ data points are underestimated in comparison with the PPD prediction. To extrapolate the value of $g_{3}\left(q^{2}\right) / g_{1}\left(q^{2}\right)$ to zero four-momentum transfer, we first adopt the monopole form, which is inspired by the PPD form. In Fig. 16, the dashed curves are fitting results by the monopole 
form with two parameters $\lambda_{3}^{(0)}$ and $\lambda_{3}^{(1)}$,

$$
\frac{g_{3}\left(q^{2}\right)}{g_{1}\left(q^{2}\right)}=\frac{\lambda_{3}^{(0)}}{1+\lambda_{3}^{(1)} q^{2}}
$$

where $1 / \sqrt{\lambda_{3}^{(1)}}$ corresponds to a monopole mass. The obtained values of $g_{3}(0) / g_{1}(0)$ are significantly smaller than those of the PPD prediction as $\left(M_{\Xi}+M_{\Sigma}\right)^{2} / M_{K}^{2}$ for the $\Xi \rightarrow \Sigma$ transition and $4 M_{N}^{2} / M_{K}^{2}$ for the $n \rightarrow p$ transition. The resulting monopole masses are also bigger than the expected Kaon and pion masses. This substantial deviation from the PPD form is mainly caused by an unexpected reduction of the lowest $q^{2}$ data points.

In Ref. [15], we have reported that the lowest $q^{2}$ point in the nucleon's induced pseudo-scalar form factor at $L=16$, which is now utilized in our current calculations, may suffer from the finite volume effect. Indeed, the $q^{2}$ dependence of the results obtained from the larger lattice $(L=24)$ was well fitted by the pion-pole structure. In this context, we may have an alternative way to evaluate the value of $g_{3}(0) / g_{1}(0)$ by a one-parameter fit in the monopole form with the monopole mass constrained by simulated $M_{K}$ or $M_{\pi}$. We simply refer to such a fit as the "Kaon-pole fit" or the "pion-pole fit" respectively. All extrapolated values of $g_{3}\left(q^{2}\right) / g_{1}\left(q^{2}\right)$ to zero four-momentum transfer with both determinations of monopole and Kaon-pole fits are listed in Table XIII.

The solid curves in Fig. 16 are given by the Kaon- and pion-pole fits. For a justification of this analysis, see the right panel of Fig. 16. In this figure, four data points (open triangles) obtained from the larger lattice $(L=24)$ are additionally included. One can easily see that these data points quite follow the solid curve, which is determined by the pion-pole fit of the lowest three $q^{2}$ data points obtained from the lattice size of $L=16$. It is found that resulting values of $g_{3}(0) / g_{1}(0)$ are still slightly smaller than the PPD values in either case of $\Xi \rightarrow \Sigma$ or $n \rightarrow p$. The similar quenching was observed in our previous detailed study of the nucleon's induced pseudo-scalar form factor using the larger lattice $(L=24)[15]$. It is worth mentioning that the size of this quenching for $\Xi \rightarrow \Sigma$ is similar to that of $n \rightarrow p$. Therefore, ratios of $\left[g_{3}(0) / g_{1}(0)\right]_{\Xi \rightarrow \Sigma}$ and $\left[g_{3}(0) / g_{1}(0)\right]_{n \rightarrow p}$ exhibit remarkable consistency with the PPD prediction as shown in Table XIV Even if we adopt the monopole form for the $q^{2}$ extrapolation, the resulting ratios still barely agree with the PPD values. Our results strongly suggest that the following relation is well fulfilled at least in the simulated region

$$
\left[\frac{g_{3}(0)}{g_{1}(0)}\right]_{\Xi \rightarrow \Sigma} \approx\left(\frac{M_{\Xi}+M_{\Sigma}}{2 M_{N}}\right)^{2} \frac{M_{\pi}^{2}}{M_{K}^{2}}\left[\frac{g_{3}(0)}{g_{1}(0)}\right]_{n \rightarrow p} .
$$

As for the chiral extrapolation of $g_{3}(0) / g_{1}(0)$, the linear fit in two mass combinations $M_{K}^{2}+M_{\pi}^{2}$ and $M_{K}^{2}-M_{\pi}^{2}$ (the same as method-B in Sec. VIB3) was utilized in Ref. [13]. However, this extrapolation doesn't take into account the expected large quark-mass dependence of $g_{3}(0) / g_{1}(0)$ in the vicinity of the chiral limit like a divergent $1 / M_{\pi}^{2}$ term for $n \rightarrow p$ or a $1 / M_{K}^{2}$ term for $\Xi \rightarrow \Sigma$. This implies that the extrapolated values should be considerably underestimated especially for the case of $n \rightarrow p$. For the ratio of $\left[g_{3}(0) / g_{1}(0)\right]_{\Xi \rightarrow \Sigma}$ and $\left[g_{3}(0) / g_{1}(0)\right]_{n \rightarrow p}$ at the physical point, the naive chiral extrapolation indeed yields a large value of $0.67(16)$ for the monopole fit or $0.52(4)$ for the Kaon(pion)-pole fit, which should be compared with the PPD value of $M_{K}^{2}\left(M_{\Xi}+M_{\Sigma}\right)^{2} /\left(4 M_{N}^{2} M_{\pi}^{2}\right)=0.1430$ at the physical point. This result is clearly contradicted with the finding expressed by Eq. (68) fulfilled in the simulated region.

The simple linear fit in two mass combinations $M_{K}^{2}+M_{\pi}^{2}$ and $M_{K}^{2}-M_{\pi}^{2}$ is instead applied to the ratio of $g_{3}(0) / g_{1}(0)$ and its PPD value, which has a very mild quark-mass dependence in either case of $\Xi \rightarrow \Sigma$ or $n \rightarrow p$. The value of $\left[g_{3}(0) / g_{1}(0)\right]_{\Xi \rightarrow \Sigma}$ at the physical point is evaluated from the extrapolated value of this ratio with a multiplicative factor of the physical PPD value. We obtain $\left[g_{3}(0) / g_{1}(0)\right]_{\Xi \rightarrow \Sigma}=21.58(98)$ and $\left[g_{3}(0) / g_{1}(0)\right]_{n \rightarrow p}=151.0(6.9)$ at the physical point for the Kaon(pion)-pole fit. The ratio of those values, which are determined to be $0.1429(2)$, correctly reproduces the PPD value. This determination is rather phenomenological. However, it is hard to perform the chiral extrapolation of $g_{3}(0) / g_{1}(0)$ without any assumption within our limited data sets. Thus, instead of quoting any final value, we would like to stress that the expected relation between $\left[g_{3}(0) / g_{1}(0)\right]_{\Xi \rightarrow \Sigma}$ and $\left[g_{3}(0) / g_{1}(0)\right]_{n \rightarrow p}$ as in Eq. (68) is confirmed in our simulations.

\section{SUMMARY}

In this paper, we have studied flavor $S U(3)$-breaking effects in the hyperon semileptonic decay, $\Xi^{0} \rightarrow \Sigma^{+} l \bar{\nu}_{l}$ using quenched DWF simulations with the lattice size $L^{3} \times T=16^{3} \times 32$. The spatial extent $L a \approx 2.4 \mathrm{fm}$ was large enough to calculate all six form factors describing the beta-decay matrix element without a serious finite volume effect on the axial-vector coupling $g_{1}(0)$. From phenomenological point of view, the significance of this subject is twofold: (1) to extract the element $V_{u s}$ of the Cabibbo-Kobayashi-Maskawa mixing matrix from the $\Delta S=1$ decay process, and 

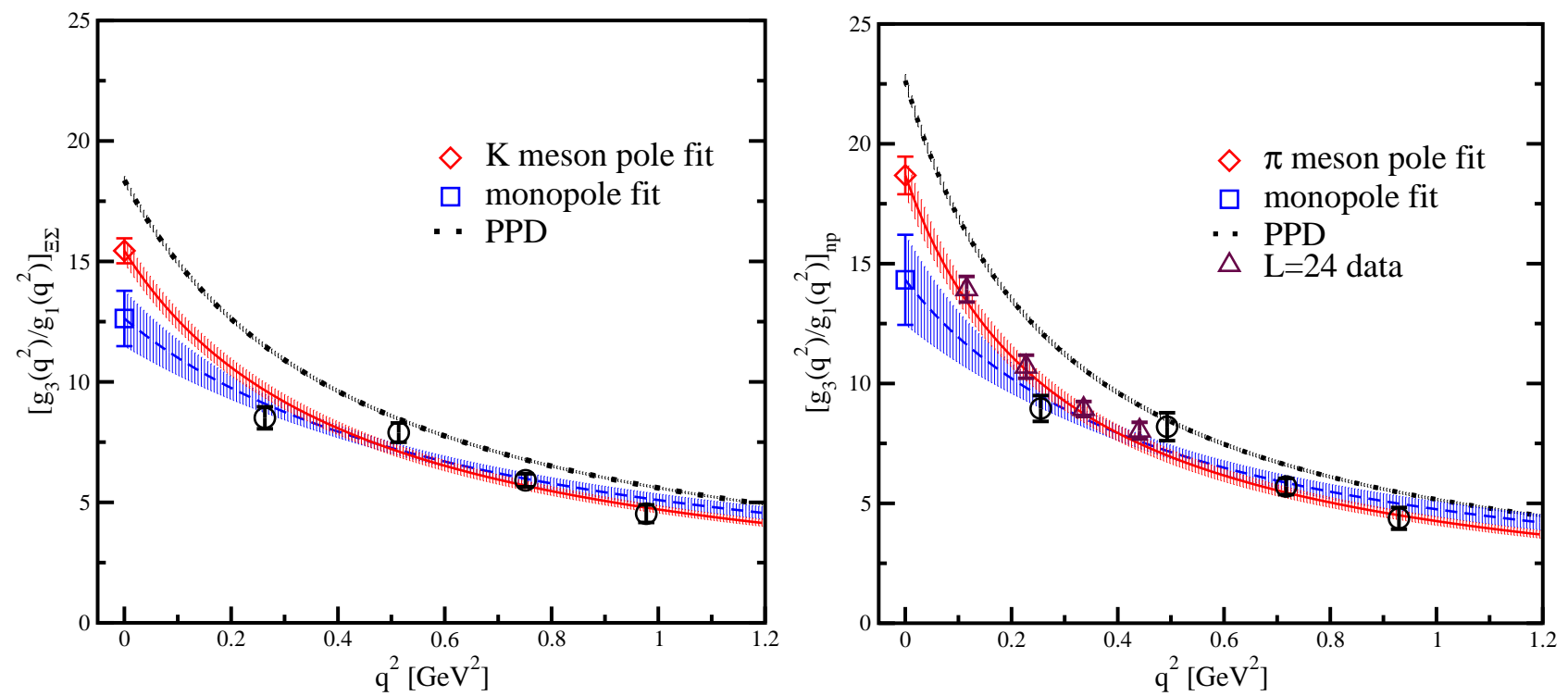

FIG. 16: The ratio $g_{3}\left(q^{2}\right) / g_{1}\left(q^{2}\right)$ as functions of four-momentum squared $q^{2}$ for $\Xi^{0} \rightarrow \Sigma^{+}$(left panel) and $n \rightarrow p$ (right panel) at $\left(m_{u d}, m_{s}\right)=(0.04,0.08)$. Symbols are defined as in Fig. 15 The highest $q^{2}$ data point is not included in our fits.

TABLE XIII: Ratio $\left[g_{3}(0) / g_{1}(0)\right]_{\Xi \rightarrow \Sigma}$ is evaluated by the $q^{2}$ extrapolation with the monopole and Kaon-pole form.

\begin{tabular}{ll|ll}
\hline \hline & & {$\left[g_{3}(0) / g_{1}(0)\right]_{\Xi \rightarrow \Sigma}$} & \\
$m_{s}$ & $m_{u d}$ & monopole & Kaon pole \\
\hline 0.08 & 0.06 & $12.08(87)$ & $14.40(42)$ \\
& 0.05 & $12.36(98)$ & $14.90(46)$ \\
& 0.04 & $12.63(1.15)$ & $15.44(52)$ \\
0.10 & 0.05 & $11.77(86)$ & $13.95(42)$ \\
& 0.04 & $11.97(1.00)$ & $14.34(47)$ \\
\hline & phys. point & $16.73(2.51)$ & $21.58(98)$ \\
\hline \hline
\end{tabular}

TABLE XIV: Comparison of measured ratios of $\left[g_{3}(0) / g_{1}(0)\right]_{\Xi \rightarrow \Sigma}$ and $\left[g_{3}(0) / g_{1}(0)\right]_{n \rightarrow p}$ to their PPD value given by $M_{K}^{2}\left(M_{\Xi}+\right.$ $\left.M_{\Sigma}\right)^{2} /\left(4 M_{N}^{2} M_{\pi}^{2}\right)$.

\begin{tabular}{ll|lll}
\hline \hline$m_{s}$ & $m_{u d}$ & From monopole fit & From Kaon(pion)-pole fit & PPD value \\
\hline 0.08 & 0.06 & $0.957(17)$ & $0.938(6)$ & $0.930(1)$ \\
& 0.05 & $0.925(33)$ & $0.891(10)$ & $0.878(2)$ \\
& 0.04 & $0.882(58)$ & $0.827(15)$ & $0.811(3)$ \\
0.10 & 0.05 & $0.881(43)$ & $0.834(14)$ & $0.819(4)$ \\
& 0.04 & $0.836(68)$ & $0.768(18)$ & $0.751(5)$ \\
\hline \hline
\end{tabular}

(2) to provide vital information to analysis of the strange quark fraction of the proton spin with the polarized deep inelastic scattering data. Our particular choice of the $\Xi^{0} \rightarrow \Sigma^{+}$decay process is highly sensitive to the flavor $S U(3)$ breaking, since this decay process is nothing but the direct analogue of neutron beta decay under the exchange of the down quark with the strange quark. The $S U(3)$-breaking pattern observed in this study is summarized in Table XV

The vector form factor at zero four-momentum transfer, $f_{1}(0)$ is protected by the Ademollo-Gatto theorem against corrections at first order in symmetry breaking. However, a sign of the second-order correction is somewhat controversial among various theoretical studies at present. Our estimate of renormalized $\left[f_{1}(0)\right]_{\Xi \rightarrow \Sigma}$ at the physical point from quenched lattice QCD simulation is $0.989(19)$, which indicates that the second-order correction on $f_{1}(0)$ is likely negative. This leads to the closer value of $\left|V_{u s}\right|$ to the value obtained from $K_{l 3}$ decays. Although both the 
TABLE XV: Summary of the $S U(3)$-breaking pattern observed in the $\Xi^{0} \rightarrow \Sigma^{+}$decay. Here, $\Delta_{S U(3)}$ is defined as $([X] \Xi \rightarrow \Sigma-$ $\left.[X]_{n \rightarrow p}\right) /[X]_{n \rightarrow p}$ for each quantity $X$. We also evaluate the scaled values of $\Delta_{S U(3)}$ by the expected size of the leading-order corrections.

\begin{tabular}{c|lll}
\hline \hline$X$ & $\Delta_{S U(3)}$ & $\Delta_{S U(3)} /(2 \delta)^{n}$ & $n$ \\
\hline$f_{1}(0)$ & $-0.013(19)$ & $-1.3(1.9)$ & 2 \\
$f_{2}(0)$ & $+0.16(11)$ & $+1.6(1.1)$ & 1 \\
$f_{3}(0) / f_{1}(0)$ & $+0.137(94)^{a}$ & $+1.4(9)$ & 1 \\
$g_{1}(0) / f_{1}(0)$ & $+0.051(35)$ & $+0.51(35)$ & 1 \\
$g_{2}(0) / g_{1}(0)$ & $+0.677(177)^{a}$ & $+6.8(1.8)$ & 1 \\
\hline \hline
\end{tabular}

${ }^{a}$ Because $f_{3}(0)=0$ and $g_{2}(0)=0$ for $n \rightarrow p,\left[f_{3}(0) / f_{1}(0)\right]_{\Xi \rightarrow \Sigma}$ and $\left[g_{2}(0) / g_{1}(0)\right]_{\Xi \rightarrow \Sigma}$ are instead quoted respectively.

$1 / N_{c}$ expansion analysis and the full one-loop $\mathcal{O}\left(p^{4}\right)$ calculation in HBChPT favor positive corrections, our observed tendency for the $S U(3)$ breaking correction agrees with predictions of quark models and CBChPT up to complete $\mathcal{O}\left(p^{4}\right)$.

The leading correction of the flavor $S U(3)$ breaking to $g_{1}(0)$ starts at first order in symmetry breaking. Although sizable breaking corrections, which is the order of $10 \%$ estimated from the mass splitting in the octet baryons, is expected, we found relatively small and positive correction to $g_{1}(0) / f_{1}(0)$ as $\left[g_{1}(0) / f_{1}(0)\right]_{\Xi \rightarrow \Sigma}=1.051(33) \times$ $\left[g_{1}(0) / f_{1}(0)\right]_{n \rightarrow p}$ in contrast to the model predictions where large and negative correction is preferable. Unfortunately, the first and single experiment of the $\Xi^{0} \rightarrow \Sigma^{+}$decay done by the $\mathrm{KTeV}$ Collaboration is not yet sufficiently precise to determine either the size, or the sign, of the $S U(3)$ breaking correction to $g_{1}(0) / f_{1}(0)$.

The advantages of lattice QCD studies of the hyperon beta decay are further demonstrated in determinations of the other beta-decay form factors, while it is difficult to determine each form factor separately in experiments. Especially, information of the second-class form factors $g_{2}$ is required since linear combinations of $g_{1}(0)$ and $g_{2}(0)$ are actually measured in the experiments from the Dalitz plot that reflects the electron-neutrino angular correlation [18]. Furthermore, the nonzero value of the weak electricity form factor $g_{2}$ as well as that of the induced scalar form factor $f_{3}$ is the direct evidence of the $S U(3)$ breaking effect in the hyperon beta decays. We obtain the ratios of $\left[g_{2}(0) / g_{1}(0)\right]_{\Xi \rightarrow \Sigma}=0.68(18)$ and $\left[f_{3}(0) / f_{1}(0)\right]_{\Xi \rightarrow \Sigma}=0.14(9)$. Although both values are roughly comparable to the expected size of the leading first-order corrections of the flavor $S U(3)$ breaking, the former is much larger than the latter.

A remarkable observation is that a relatively small first-order correction to $g_{1}(0)$ is accidentally compensated for flavor $S U(3)$-breaking effects on $\tilde{g}_{1}(0)$ with an opposite contribution due to the relatively large and positive value of $g_{2}(0)$. This may suggest why the conventional analysis of the hyperon beta decays based on the Cabibbo hypothesis works well, though the effects due to the $S U(3)$ breaking observed in the octet baryon masses and magnetic moments are expected to considerably affect the axial-vector part of the weak matrix elements.

We have also found that the weak magnetism $f_{2}(0)$ receives positive corrections of order $16 \%$ for the flavor $S U(3)$ breaking, measuring $\left[f_{2}(0) / f_{1}(0)\right]_{\Xi \rightarrow \Sigma}=1.16(10) \times\left[f_{2}(0) / f_{1}(0)\right]_{n \rightarrow p}$. Our result is not in agreement with either the generalized CVC hypothesis or the Cabibbo-model prediction. On the other hand, as for the induced pseudo-scalar form factor $g_{3}$, the generalized PPD form, which is extended even in $\Delta S=1$ decays under the strong assumption of PCAC, provides a good prediction of the ratio of $\left[g_{3}(0) / g_{1}(0)\right]_{\Xi \rightarrow \Sigma}$ and $\left[g_{3}(0) / g_{1}(0)\right]_{n \rightarrow p}$ at the physical point as $M_{K}^{2}\left(M_{\Xi}+M_{\Sigma}\right)^{2} /\left(4 M_{N}^{2} M_{\pi}^{2}\right)=0.1430$. This indicates that the large $S U(3)$-breaking effects on $g_{3}(0) / g_{1}(0)$ is attributed to the Kaon(pion)-pole structure of the $g_{3}$ form factor.

In this study, we have focused only on the specific beta-decay process, $\Xi^{0} \rightarrow \Sigma^{+}$, However, if the flavor $S U(3)$ breaking pattern observed here would commonly appear in other beta-decay processes, our results call for nonnegligible $S U(3)$-breaking effects in all hyperon beta decays. We also believe that the quenched approximation is not problematic for the determination of flavor $S U(3)$-breaking effects in the hyperon beta decays in similar to what was observed in calculations of $K_{l 3}$ decays [1, 2, 3]. Nevertheless, the simulation with dynamical $2+1$ flavor quarks is an important future direction to be explored for full knowledge of the $S U(3)$-breaking pattern in the hyperon beta decays. Especially, in order to settle the signs of the leading order correction on $f_{1}(0), f_{2}(0)$ and $g_{1}(0) / f_{1}(0)$, more extensive lattice study is required. We plan to extend the present calculation to include other relevant hyperon beta-decay processes such as $\Sigma^{-} \rightarrow n$ and $\Lambda \rightarrow p$ using dynamical $N_{f}=2+1$ flavor DWF lattice configurations generated by the RBC and UKQCD Collaborations [53, 54]. Such planning is now underway [55] 


\section{Appendix A: Scalar function $f_{S}$}

Let us consider the matrix element of the divergence of the vector current:

$$
\begin{aligned}
\left\langle b\left(p^{\prime}\right)\left|\partial_{\alpha} V_{\alpha}(0)\right| B(p)\right\rangle & =\bar{u}_{b}\left(p^{\prime}\right)\left[i\left(p-\not p^{\prime}\right) f_{1}^{B \rightarrow b}\left(q^{2}\right)-\frac{q^{2}}{M_{B}+M_{b}} f_{3}^{B \rightarrow b}\left(q^{2}\right)\right] u_{B}(p) \\
& =\left[\left(M_{b}-M_{B}\right) f_{1}^{B \rightarrow b}\left(q^{2}\right)-\frac{q^{2}}{M_{B}+M_{b}} f_{3}^{B \rightarrow b}\left(q^{2}\right)\right] \bar{u}_{b}\left(p^{\prime}\right) u_{B}(p) .
\end{aligned}
$$

Here, we have used the Dirac equation for both initial $(B)$ and final $(b)$ baryon states, $\left(i p p+M_{B}\right) u_{B}(p)=\bar{u}_{b}\left(p^{\prime}\right)\left(i \not p^{\prime}+\right.$ $\left.M_{b}\right)=0$ to get from the first line to the second line. Combined with Eq. (44), one finds the following relation

$$
\left\langle b\left(p^{\prime}\right)\left|\partial_{\alpha} V_{\alpha}(0)\right| B(p)\right\rangle=\left(M_{b}-M_{B}\right) f_{S}^{B \rightarrow b}\left(q^{2}\right) \bar{u}_{b}\left(p^{\prime}\right) u_{B}(p),
$$

where an overall factor $M_{b}-M_{B}$ on the right hand side is responsible for the current conservation when the flavor $S U(3)$ symmetry is exact $\left(M_{b}=M_{B}\right)$.

\section{Appendix B: Other parametrization of the baryon weak matrix element}

Instead of the standard parametrization of Eqs. (2) and (3), the following equivalent form [56] is more useful to derive all of Eqs. (15)-(19), which are considered at the rest flame of the final $(b)$ state $\left(\mathbf{p}^{\prime}=\mathbf{0}\right)$ :

$$
\begin{aligned}
& \mathcal{O}_{\alpha}^{V}(q)=\gamma_{\alpha} \tilde{f}_{1}^{B \rightarrow b}\left(q^{2}\right)+i p_{\alpha} \frac{\tilde{f}_{2}^{B \rightarrow b}\left(q^{2}\right)}{M_{B}+M_{b}}+i q_{\alpha} \frac{\tilde{f}_{3}^{B \rightarrow b}\left(q^{2}\right)}{M_{B}+M_{b}}, \\
& \mathcal{O}_{\alpha}^{A}(q)=\gamma_{\alpha} \gamma_{5} \tilde{g}_{1}^{B \rightarrow b}\left(q^{2}\right)+i p_{\alpha} \gamma_{5} \frac{\tilde{g}_{2}^{B \rightarrow b}\left(q^{2}\right)}{M_{B}+M_{b}}+i q_{\alpha} \gamma_{5} \frac{\tilde{g}_{3}^{B \rightarrow b}\left(q^{2}\right)}{M_{B}+M_{b}} .
\end{aligned}
$$

The two sets of form factors are connected by

$$
\begin{array}{ll}
\tilde{f}_{1}^{B \rightarrow b}\left(q^{2}\right)=f_{1}^{B \rightarrow b}\left(q^{2}\right)+f_{2}^{B \rightarrow b}\left(q^{2}\right), & \tilde{g}_{1}^{B \rightarrow b}\left(q^{2}\right)=g_{1}^{B \rightarrow b}\left(q^{2}\right)-\frac{M_{B}-M_{b}}{M_{B}+M_{b}} g_{2}^{B \rightarrow b}\left(q^{2}\right), \\
\tilde{f}_{2}^{B \rightarrow b}\left(q^{2}\right)=2 f_{2}^{B \rightarrow b}\left(q^{2}\right), & \tilde{g}_{2}^{B \rightarrow b}\left(q^{2}\right)=2 g_{2}^{B \rightarrow b}\left(q^{2}\right), \\
\tilde{f}_{3}^{B \rightarrow b}\left(q^{2}\right)=f_{3}^{B \rightarrow b}\left(q^{2}\right)-f_{2}^{B \rightarrow b}\left(q^{2}\right), & \tilde{g}_{3}^{B \rightarrow b}\left(q^{2}\right)=g_{3}^{B \rightarrow b}\left(q^{2}\right)-g_{2}^{B \rightarrow b}\left(q^{2}\right),
\end{array}
$$

One can easily check above relations using the Gordon identity.

\section{Acknowledgments}

We would like to thank our colleagues in the RBC collaboration and especially T. Blum for helpful suggestions. We also thank RIKEN, Brookhaven National Laboratory and the U.S. DOE for providing the facilities essential for the completion of this work. The results of calculations were performed by using of QCDOC at RIKEN BNL Research Center. S.S. is supported by the JSPS for a Grant-in-Aid for Scientific Research (C) (No. 19540265). T.Y. was supported by the U.S. DOE under contract DE-FG02-92ER40716, and is the Yukawa Fellow supported by Yukawa Memorial Foundation.

[1] P. A. Boyle et al., Phys. Rev. Lett. 100, 141601 (2008).

[2] D. Becirevic et al., Nucl. Phys. B 705, 339 (2005).

[3] C. Dawson, T. Izubuchi, T. Kaneko, S. Sasaki and A. Soni, Phys. Rev. D 74, 114502 (2006).

[4] N. Cabibbo, Phys. Rev. Lett. 10, 531 (1963).

[5] M. Kobayashi and T. Maskawa, Prog. Theor. Phys. 49, 652 (1973).

[6] W. J. Marciano, PoS KAON, 003 (2008).

[7] H. Leutwyler and M. Roos, Z. Phys. C 25, 91 (1984).

[8] V. Mateu and A. Pich, JHEP 0510, 041 (2005).

[9] For a review of hyperon beta decays, see N. Cabibbo, E. C. Swallow and R. Winston, Ann. Rev. Nucl. Part. Sci. 53, 39 (2003) and references therein. 
[10] For a review of spin physics, see B. W. Filippone and X. D. Ji, Adv. Nucl. Phys. 26, 1 (2002).

[11] For a recent review, see P. G. Ratcliffe, Czech. J. Phys. 54, B11 (2004).

[12] T. Yamanishi, Phys. Rev. D 76, 014006 (2007).

[13] D. Guadagnoli, V. Lubicz, M. Papinutto and S. Simula, Nucl. Phys. B 761, 63 (2007).

[14] S. Sasaki, K. Orginos, S. Ohta and T. Blum, Phys. Rev. D 68, 054509 (2003).

[15] S. Sasaki and T. Yamazaki, Phys. Rev. D 78, 014510 (2008).

[16] A. Alavi-Harati et al. [KTeV Collaboration], Phys. Rev. Lett. 87, 132001 (2001).

[17] S. Weinberg, Phys. Rev. 112, 1375 (1958).

[18] J. M. Gaillard and G. Sauvage, Ann. Rev. Nucl. Part. Sci. 34, 351 (1984).

[19] M. Ademollo and R. Gatto, Phys. Rev. Lett. 13, 264 (1964).

[20] J. F. Donoghue and B. R. Holstein, Phys. Rev. D 25, 206 (1982).

[21] J. F. Donoghue, B. R. Holstein and S. W. Klimt, Phys. Rev. D 35, 934 (1987).

[22] F. Schlumpf, Phys. Rev. D 51, 2262 (1995).

[23] R. Flores-Mendieta, E. Jenkins and A. V. Manohar, Phys. Rev. D 58, 094028 (1998).

[24] G. Villadoro, Phys. Rev. D 74, 014018 (2006).

[25] A. Lacour, B. Kubis and U. G. Meissner, JHEP 0710, 083 (2007).

[26] P. G. Ratcliffe, Phys. Rev. D 59, 014038 (1998).

[27] C. Amsler et al. [Particle Data Group], Phys. Lett. B 667, 1 (2008).

[28] A. Sirlin, Nucl. Phys. B 161, 301 (1979).

[29] T. Takaishi, Phys. Rev. D 54, 1050 (1996).

[30] P. de Forcrand et al. [QCD-TARO Collaboration], Nucl. Phys. B 577, 263 (2000).

[31] Y. Aoki et al., Phys. Rev. D 69, 074504 (2004).

[32] H. W. Lin, T. Blum, S. Ohta, S. Sasaki and T. Yamazaki, Phys. Rev. D 78, 014505 (2008)

[33] T. Yamazaki et al. [RBC+UKQCD Collaboration], Phys. Rev. Lett. 100, 171602 (2008).

[34] Y. Aoki et al., Phys. Rev. D 73, 094507 (2006).

[35] Y. Aoki, C. Dawson, J. Noaki and A. Soni, Phys. Rev. D 75, 014507 (2007).

[36] S. Sasaki and T. Yamazaki, PoS LAT2006, 092 (2006), arXiv:hep-lat/0610082.

[37] S. Gusken, Nucl. Phys. Proc. Suppl. 17 (1990) 361.

[38] C. Alexandrou, S. Gusken, F. Jegerlehner, K. Schilling and R. Sommer, Nucl. Phys. B 414, 815 (1994).

[39] F. Berruto, T. Blum, K. Orginos and A. Soni, Phys. Rev. D 73, 054509 (2006).

[40] S. Sasaki, T. Blum and S. Ohta, Phys. Rev. D 65, 074503 (2002).

[41] K. Sasaki and S. Sasaki, Phys. Rev. D 72, 034502 (2005)

[42] P. Hägler et al., [LHPC collaboration], Phys. Rev. D 68, 034505 (2003).

[43] D. B. Kaplan, Phys. Lett. B 288, 342 (1992).

[44] Y. Shamir, Nucl. Phys. B 406, 90 (1993).

[45] V. Furman and Y. Shamir, Nucl. Phys. B 439, 54 (1995).

[46] T. Blum et al., Phys. Rev. D 66, 014504 (2002).

[47] N. Yamada, S. Aoki and Y. Kuramashi, Nucl. Phys. B 713, 407 (2005).

[48] S. Hashimoto, A. X. El-Khadra, A. S. Kronfeld, P. B. Mackenzie, S. M. Ryan and J. N. Simone, Phys. Rev. D 61, 014502 (1999).

[49] Y. Nambu, Phys. Rev. Lett. 4, 380 (1960).

[50] S. Choi et al., Phys. Rev. Lett. 71, 3927 (1993).

[51] T. Gorringe and H. W. Fearing, Rev. Mod. Phys. 76, 31 (2003).

[52] W. I. Weisberger, Phys. Rev. 143, 1302 (1966).

[53] C. Allton et al. [RBC and UKQCD Collaborations], Phys. Rev. D 76, 014504 (2007).

[54] C. Allton et al. [RBC-UKQCD Collaboration], Phys. Rev. D 78, 114509 (2008).

[55] S. Sasaki and T. Yamazaki (in progress).

[56] V. Linke, Nucl. Phys. B 12, 669 (1969).

[57] Their obtained value of $\left[g_{1}(0) / f_{1}(0)\right]_{\Sigma \rightarrow n}=-0.287(52)$ is rather large compared to the experimental value, $-0.340(17)$ 9]. This discrepancy could be attributed to the serious finite volume effect on the axial-vector coupling $g_{1}(0)$, which was observed in the case of neutron beta decay. Note that they utilized about $(1.8 \mathrm{fm})^{3}$ volume, which is not enough large at least for neutron beta decay [14, 15].

[58] Remark that our $\gamma_{5}$ definition $\gamma_{5} \equiv \gamma_{x} \gamma_{y} \gamma_{z} \gamma_{t}=-\gamma_{5}^{M}$ has the opposite sign relative to that in the Minkowski convention $\left(\vec{\gamma}^{M}=i \vec{\gamma}\right.$ and $\left.\gamma_{0}^{M}=\gamma_{t}\right)$ adopted in the particle data group. In addition, $q^{2}$ denoted in this paper, which stands for Euclidean four-momentum squared, corresponds to the timelike momentum squared as $q_{M}^{2}=-q^{2}<0$ in Minkowski space.

[59] Note that in our convention the time-reversal process, $b \rightarrow B$, gives the relation for the first-class form factors $f_{1,2}^{B \rightarrow b}\left(q^{2}\right)=$ $f_{1,2}^{b \rightarrow B}\left(q^{2}\right)$ and $g_{1,3}^{B \rightarrow b}\left(q^{2}\right)=g_{1,3}^{b \rightarrow B}\left(q^{2}\right)$, while $f_{3}^{B \rightarrow b}\left(q^{2}\right)=-f_{3}^{b \rightarrow B}\left(q^{2}\right)$ and $g_{2}^{B \rightarrow b}\left(q^{2}\right)=-g_{2}^{b \rightarrow B}\left(q^{2}\right)$ for the first-class form factors 17 .

[60] In this paper, we restrict ourselves to considering the iso-spin symmetric case as $m_{u d}=m_{u}=m_{d}$

[61] In our actual simulations, the time-reversal process, $\Sigma^{+} \rightarrow \Xi^{0}$, was actually utilized. This gives us some initial confusion in data analysis of Ref. [36]. We now update all of results in this paper.

[62] There is no differences in the final results between using the dispersion relation, and the fitted energies at non-zero momenta within statistical errors, while the former statistical errors are slightly smaller than the latter. 
[63] Here, we should comment that we did not use the double ratio method, which is originally proposed in Ref. [48] for $B$ meson decays and also adopted in Ref. 13] for the hyperon beta decays.

[64] Strictly speaking, it is true only if the spatial momentum transfer q satisfies the condition $\mathbf{q}^{2}<\left(M_{B}^{2}-M_{b}^{2}\right)^{2} /\left(4 M_{b}^{2}\right)$. Our accessible finite momentum is much larger than $\left(M_{B}^{2}-M_{b}^{2}\right)^{2} /\left(4 M_{b}^{2}\right)$ in this calculation.

[65] Even the physical value of $q_{\max }^{2}$ is relatively close to $q^{2}=0$ in the case of the hyperon beta decays

[66] It should be reminded that the value of $\delta$ for $\Xi \rightarrow \Sigma$ at the physical point is 0.04996 , which is indeed small. Here, we evaluate $\delta$ with $M_{\Sigma}=1193.2 \mathrm{MeV}$ and $M_{\Xi}=1318.7 \mathrm{MeV}$, which are the isospin-averaged masses.

[67] In the quantitative sense, the size of $2 \delta$ is more relevant than the size of $\delta$ for quoting the real size of the flavor $S U(3)$ breaking.

[68] In the case of $\mathbf{n}^{2}=3$, we use Eq. (52) for evaluate $\Lambda_{L}^{A, B \rightarrow b}\left(q_{z}=0\right)$.

[69] We found an error in the third term of Eq. (20) in Ref. [13]. Note that we formulate both of Eqs. (45)-(47) and Eqs. (49)-(51) in a fully covariant way, while only a single component of the Dirac indices is considered in Ref. [13].

[70] We observe that this difference becomes very small at a finer lattice spacing, $a \approx 0.11 \mathrm{fm}$, in dynamical $N_{f}=2+1$ flavor DWF calculations [55].

[71] The subtraction procedure is not unique. Alternatively, we may consider a simple difference of measured second-class form factors between $\Xi \rightarrow \Sigma$ and $n \rightarrow p$ processes as $\left[f_{3}^{\text {sub }}\left(q^{2}\right)\right]_{\Xi \rightarrow \Sigma}=\left[f_{3}^{\text {sub }}\left(q^{2}\right)\right]_{\Xi \rightarrow \Sigma}-\left[f_{3}^{\text {sub }}\left(q^{2}\right)\right]_{n \rightarrow p}$. The leading-order behavior of the flavor $S U(3)$ breaking in the second-class form factors is not changed by a variation of the subtraction term. The resulting difference appears only in higher-order corrections. Indeed, analyses with two types of the subtraction term yield fairly consistent results of $f_{3}(0)$ and $g_{2}(0)$ at the physical point.

[72] If we consider inclusion of either $\left(M_{K}^{2}+M_{\pi}^{2}\right)^{2}$ or $M_{K}^{2}-M_{\pi}^{2}$ terms into Eq. (57), our limited data points can not sufficiently determine either coefficients, which receive more than $100 \%$ error. The resulting values at the physical point are consistent with the results obtained from the simple linear fit (57) within the statistical errors.

[73] A factor $\left(M_{\Xi}+M_{\Sigma}\right) / M_{\Xi}$, equal to $\simeq 1.9048$ is different from definitions of $f_{3}$ and $g_{2}$ form factors adopted in Ref. [16].

[74] Note that the presence of the second-class form factor $g_{2}$ does not modify the generalized Goldberger-Treiman relation [52], which is essential for the applicability of the PPD form [15]. 